\title{
TUTORIAL
}

\section{The dynamic neural field approach to cognitive robotics*}

\author{
Wolfram Erlhagen $^{1}$ and Estela Bicho ${ }^{2}$ \\ ${ }^{1}$ Department of Mathematics for Science and Technology, Universidade do Minho, \\ 4800-058 Guimarães, Portugal \\ ${ }^{2}$ Department of Industrial Electronics, Universidade do Minho, 4800-058 Guimarães, Portugal \\ E-mail: wolfram.erlhagen@mct.uminho.pt
}

Received 15 December 2005

Accepted for publication 10 May 2006

Published 27 June 2006

Online at stacks.iop.org/JNE/3/R36

\begin{abstract}
This tutorial presents an architecture for autonomous robots to generate behavior in joint action tasks. To efficiently interact with another agent in solving a mutual task, a robot should be endowed with cognitive skills such as memory, decision making, action understanding and prediction. The proposed architecture is strongly inspired by our current understanding of the processing principles and the neuronal circuitry underlying these functionalities in the primate brain. As a mathematical framework, we use a coupled system of dynamic neural fields, each representing the basic functionality of neuronal populations in different brain areas. It implements goal-directed behavior in joint action as a continuous process that builds on the interpretation of observed movements in terms of the partner's action goal. We validate the architecture in two experimental paradigms: (1) a joint search task; (2) a reproduction of an observed or inferred end state of a grasping-placing sequence. We also review some of the mathematical results about dynamic neural fields that are important for the implementation work.
\end{abstract}

(Some figures in this article are in colour only in the electronic version)

\section{Introduction}

Research in autonomous robotics has shown that fairly complex robot behaviors may be organized without the need for high-level world representations which are central to the traditional artificial intelligence approach (for a review see, e.g., Arkin (1998)). The architectures for autonomous robots are built on the idea that 'autonomous agents' should structure their behaviors on the basis of sensory information that they themselves acquire. The closed loop between sensors and effectors enables a fast real-time adaptation to changing conditions in dynamic and a priori unknown environments. Entire chains of behaviors may emerge through learned

* Part of the 3rd Neuro-IT and Neuroengineering Summer School Tutorial Series. interaction among individual behaviors. Thus far, most of the applications in robotics have focused on sensory-motor tasks such as navigation, walking or object manipulation.

A major challenge for sensor-near control architectures is that so much of what defines an adequate action in more cognitive tasks depends on past and future states of the environment that are not represented in the immediate stream of sensory signals. Imagine, for instance, a joint action task in which two robots have to search and subsequently transport particular objects to a predefined area of their workspace. To guarantee for an efficient team strategy, the robots should have the capacity to anticipate the consequences of an ongoing action displayed by the partner. This implies the capacity to understand the observed movements as directed towards a specific goal (e.g., grasping a particular object). Given that in cluttered and dynamic environments occluding surfaces often disrupt the spatiotemporal continuity of the sensory input, the 
prediction may be possible only by integrating memorized information or additional contextual cues. The reasoning about the partner's current action goal should then bias the decision process about the object which will be grasped next. Cognitive functions such as memorizing, decision making, prediction and action understanding are thus essential for the collaborative task execution.

Our attempt to implement these capacities in autonomous robots is guided by the hypothesis that we can learn from the processing principles in biological nervous systems. There are three main ideas that have inspired our work. First, a growing body of neurophysiological evidence supports the existence of neural populations in different brain areas implementing the process of simulating consequences of perceptual and motor events. These internal representations are still close to the sensors and effectors since they are part of the neural circuitry which becomes active during normal perception and action.

An example that has attracted a lot of attention also in robotics over the last couple of years is the existence of the so-called mirror neurons in the premotor cortex (Rizzolatti et al 2001, Schaal 1999). These neurons respond both when the monkey makes a goal-directed action such as grasping an object and when it observes another individual performing a similar action. Mirror neurons may thus constitute the neural correlate for a matching of action observation and action execution which may account for our everyday ability to make sense of the motor behavior of others.

Another example for a neural representation consistent with the simulation idea is a class of cells recently described in the visual motion area STS (superior temporal sulcus). They seem to encode the position of an individual disappearing behind an occluding surface. These cells may thus contribute to an abstract conceptual representation of object permanence (Baker et al 2001, Jellema and Perrett 2002).

A second characteristic property of neural circuits is the ability to transiently hold and manipulate goal-related information to guide forthcoming actions. Persistent activity in specific subpopulations which carry the information about previously encountered stimuli has been observed in a wide variety of brain areas (for a review see Brody et al (2003)). Neurocomputational models of this type of shortterm memory suggest that the activity is sustained through recurrent excitatory interactions in pools of neurons that are counterbalanced by inhibitory feedback loops (Dusterwitz et al 2000). The existence of persistent activity in the brain appears to be closely related to the formation of categorical decisions (Desimone and Duncan 1995). Imagine, for instance, a network encoding object position. If the recurrent inhibition within the network is sufficiently strong, the network supports only a single localized activity pattern representing the selected target even if the bottom-up input indicates the presence of several objects in the scene.

A third idea concerns the integration of task-relevant information over time. The basic hypothesis is that the accumulation of sensory and other evidence leads to a continuous increase of the population activity representing the decision variable. When a certain threshold value is reached, the integration process is over and a decision is made which is typically linked to behavior (e.g., grasping the selected target). The underlying neural circuits seem to be sensitive to a host of psychological factors (Gold and Shadlen 2002). Neural populations in various brain areas known to be involved in organizing forthcoming actions selectively increase their baseline activity in response to precued task information or prior probabilities accumulated from past experiments (Bastian et al 2003, Asaad et al 2000). The integration of additional information sources including input from simulation loops brings neuronal population closer to the threshold level and thus biases the decision processes. This neuronal mechanism is thought to underlie the remarkable capacity of the mammalian brain to quasi-instantaneously adapt behavior to the context of sensory signals (Salinas 2003).

In this tutorial, we illustrate our attempt to endow autonomous robots with some cognitive abilities using two examples of a joint action task. The first paradigm is the above-mentioned joint search which allows us to show the impact of memory and prediction on behavior. The second task is basically a grasping-placing sequence, constrained by an obstacle, in which objects have to be placed at specific goal positions. We use this task in an imitation paradigm in which a robot has to reproduce the observed or inferred end state of a sequence displayed by a human model. Here, the emphasis is on action understanding through motor simulation and skill transfer from an experienced teacher.

The two examples illustrate our general research goal to allow robotic systems to interact with humans and other robots in a natural and useful way.

As a design tool and mathematical language for implementing the cognitive functions in our robots, we apply the framework of dynamic neural fields (DNFs) that was originally proposed to explain pattern formation in neural tissue (Wilson and Cowan 1973, Amari 1977). In the domain of robotics, DNFs have been first introduced by Schöner et al (1995) (for follow-up studies see Engels and Schöner (1995), Bergener et al (1999), Bicho et al (2000), Menzner et al (2000), Quoy et al (2003) and Erlhagen et al (2006b)) to endow the so-called dynamic approach to robotics with representation and memory. The authors demonstrated the advantages in a navigation and obstacle avoidance task. The links to neurobiology were addressed on a rather conceptual level stressing the idea that neural information processing takes place in the form of self-stabilized activation patterns.

Following this general idea, the main goal of this tutorial is to make the link to neurobiology more specific. Over the last 15 years or so, a large number of neuroimaging and electrophysiological techniques have been used to study in humans and monkeys the neural mechanisms of social cognition. New insights into the functional and anatomical organization of specific neural circuits have inspired our robot control architecture for the joint action tasks. The core part of the architecture consists of several interconnected DNFs which represent the basic functionality of neuronal populations in distinct but anatomically connected brain areas. The internal dynamics of this system evolve under the influence of external visual input and provides the decision variables for a coherent motor behavior of the robots. It is important to stress 


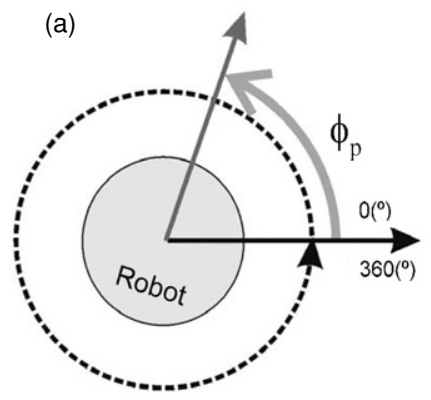

(b)

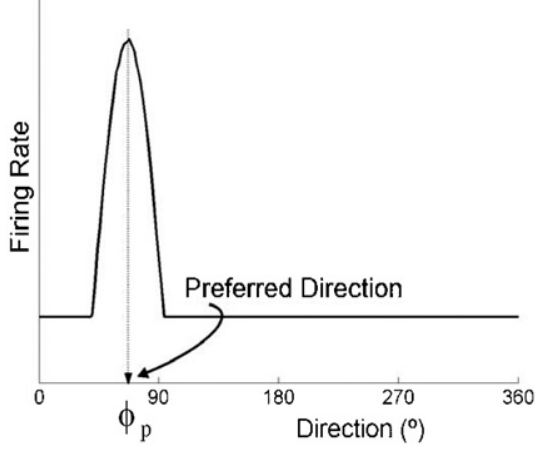

Figure 1. (a) Schematic view of the heading direction $\phi$ of the robot with respect to a fixed frame of reference. (b) Localized firing pattern of neurons encoding the direction $\phi_{p}$.

that our cognitive neuroscience-oriented architecture contrasts to the sense-think-act cycle of the traditional information processing approach (for a discussion see Pfeifer and Scheier (1999)). The experimental findings suggest that perceptual and action processes share a common neural substrate and are functionally intertwined: perception serves action but action is also a means to perception (Jackson and Decety 2004). It is believed that motor representations may play an important role in the processing of sensory information.

To illustrate the architecture and dynamics of the field model that controls the behavior of the robots in the joint action tasks, we use a simulator environment. We only briefly discuss aspects of the implementation in real robots and refer for a more detailed description to the relevant publications (e.g., Bicho et al (2000), Bicho (2000), Erlhagen et al (2006b)).

This tutorial is organized as follows. In section 2, we give an overview about some mathematical results concerning dynamic neural fields which are relevant for the implementation work. A discussion of recent neurophysiological results is presented in section 3 together with a demonstration of the approach in the two joint action tasks. We finish with a discussion of extensions and the relation to other modeling and robotics work.

\section{Dynamic neural fields}

Based on the anatomical fact that the largest input to cortical cells comes from neighboring excitatory cells, it has been suggested that recurrent interactions in populations of neurons form the basic mechanism for cortical information processing (Douglas et al 1995). The recurrent cortical architecture is believed to amplify and stabilize noisy or corrupted feed forward input signals. Moreover, when information is sustained internally in the brain by means of persistent neural activity patterns, it may be used to guide complex behavior which goes beyond a simple input-output information processing scheme. In their pioneering work, Wilson and Cowan (1973) and Amari (1977) have proposed dynamic neural fields as a simplified mathematical model for neural processing based on recurrent interactions. These models neglect the temporal dynamics of individual neurons but use instead the averaged firing rate as a means to transmit information. In the following, we summarize the properties of the particular DNF model proposed by Amari which we use in the robotic applications. The Amari model is particularly appealing since it allows under some simplifying assumptions for a complete analytical description of the dynamics of pattern formation. As a concrete and experimentally welldocumented example, we use the cortical representation of the variable direction with respect to a fixed frame of reference (figure 1(a)). The so-called head-direction (HD) cells originally found in freely moving rats (for a review see Taube and Bassett (2003)) encode the instantaneous head direction of the animal in the horizontal plane. The frame of reference is world centered so that HD cells may serve as a type of neural compass no matter where the animal is located. Once activated they maintain their firing pattern even in total darkness with all external inputs removed. An individual HD cell has its maximum firing rate at only one particular direction and firing rates decrease monotonically on either side moving away from this 'preferred direction'. For a whole population of HD cells with all directions equally represented, this means that a particular head direction is represented by a stable and localized activity profile or pulse in parametric space (figure 1(b)).

\subsection{Stationary pulse solutions}

The Amari model explains the existence of such a pattern as the result of a cortical circuitry acting in a center-surround fashion. The model network contains a circular field of interacting HD cells with an interaction strength, $w\left(\phi, \phi^{\prime}\right)$, between any two neurons $\phi$ and $\phi^{\prime}$ which depends only on the distance (i.e., $\left.\Delta \phi=\left|\phi-\phi^{\prime}\right|\right)$ of their preferred directions. It is assumed that nearby cells excite each other, whereas separated pairs of cells have a mutually inhibitory influence. Figure 2(a) depicts an example of such a synaptic weight function given by a Gaussian minus a constant $w_{\text {inhib }}>0$ :

$$
w\left(\phi-\phi^{\prime}\right)=A \exp \left(-\left(\phi-\phi^{\prime}\right)^{2} / 2 \sigma^{2}\right)-w_{\text {inhib }},
$$

where $A>0$ and $\sigma>0$ describe the amplitude and the standard deviation, respectively.

Let $u(\phi, t)$ denote the synaptic input or activation at time $t$ to a neuron encoding direction $\phi$. This activation evolves 

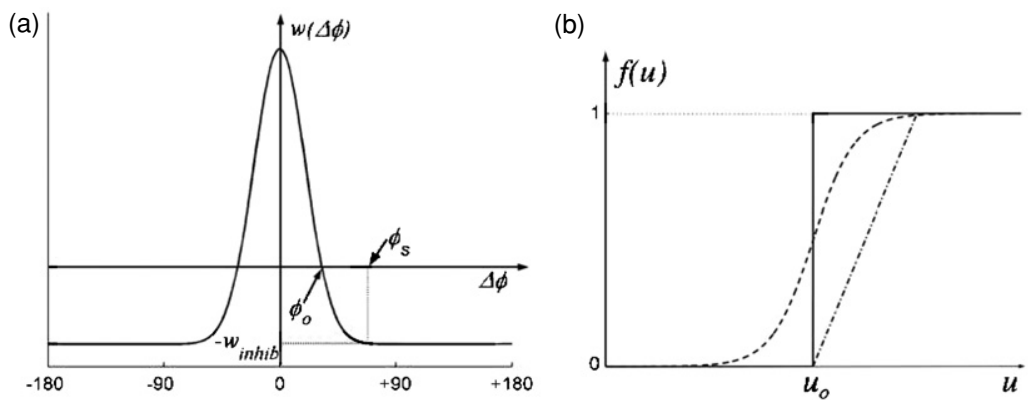

Figure 2. (a) Symmetric synaptic weight function $w(\Delta \phi), \Delta \phi=\phi-\phi^{\prime}$, of center-surround type. The synaptic weights are positive ('excitatory') for two cells $\phi$ and $\phi^{\prime}$ that are closer to each other than the distance $\phi_{0}$ and are negative ('inhibitory') for larger distances. For $\Delta \phi>\phi_{s}$, inhibition strength is constant $\left(=-w_{\text {inhib }}\right)$. (b) Three different output functions with threshold $u_{0}$ are shown: sigmoid function (dashed line), step function (solid line), ramp function (dash-dotted line).

in time as a function of external stimulation and input from other neurons. A large number of interacting neurons create a network that is almost homogeneous along the cortical surface and can thus be approximated as a continuous field of neural activation. The field dynamic is governed by the following nonlinear integro-differential equation:

$$
\begin{aligned}
& \tau \frac{\delta u(\phi, t)}{\delta t}=-u(\phi, t)+S(\phi, t) \\
& \quad+\int_{0}^{360} w\left(\phi-\phi^{\prime}\right) f\left(u\left(\phi^{\prime}, t\right)\right) \mathrm{d} \phi^{\prime}+h .
\end{aligned}
$$

Parameter $\tau>0$ defines the time scale of the field. $S(\phi, t)$ is external input at time $t$ to neuron encoding direction $\phi$. It is excitatory $(S(\phi, t)>0)$ when direction $\phi$ is specified by external information and $S(\phi, t) \leqslant 0$ otherwise.

Without the integral term, i.e.,

$$
\tau \frac{\delta u(\phi, t)}{\delta t}=-u(\phi, t)+S(\phi, t)+h,
$$

the field activity would simply relax to $S(\phi, t)+h$, with a lowpass characteristic. This dynamic is not yet sufficient by itself, as it merely reproduces the shape of the input pattern. What we need, as described above, are mechanisms that activate only one pulse (i.e., a localized pattern of activation as depicted in figure 1(b)) if multiple pools of neurons receive similar or even identical input (selection), and that are capable of retaining activation after external input is removed (memory). Clearly, such mechanisms involve interaction, that is, the evolution of activation at one location of the field depends on the level of activation at other locations of the field. The integral term describes the interaction process as a weighted summation of activity from neighboring field locations. The output function $f(u)$ gives the firing rate of a neuron with activation $u$. It is a monotonically increasing nonlinear function saturating to a constant for large $u$. To simplify the analytical work, Amari assumed that $f$ is the Heaviside function $H_{u_{0}}$ with threshold $u_{0}=0$ (figure 2(b)):

$$
H_{u_{0}}(u)= \begin{cases}0, & u<u_{0}, \\ 1, & u \geqslant u_{0} .\end{cases}
$$

However, this function is not continuously differentiable which makes it inappropriate for certain applications. One can also use other nonlinear functions which describe a more gradual increase of the firing rate (which also seems more appropriate with respect to biology), such as the sigmoid function with slope parameter $\beta$ (figure 2(b))

$$
f(u)=\frac{1}{1+\exp \left(-\beta\left(u-u_{0}\right)\right)}
$$

or the ramp function (figure 2(b))

$$
f(u)= \begin{cases}0, & u<u_{0}, \\ \beta\left(u-u_{0}\right), & u_{0} \leqslant u<u_{0}+1 / \beta, \\ 1, & u \geqslant u_{0}+1 / \beta .\end{cases}
$$

Amari's (1977) main results concerning the existence and stability of stationary solutions of (2) carry over to the case where $f$ is of the more general sigmoid type because the neural field solutions are structurally stable (Kishimoto and Amari 1979).

2.1.1. Equilibrium solutions in the absence of external input. For $S(\phi, t) \equiv 0$, equilibrium solutions ${ }^{3}$ of equation (2) are given by

$$
u(\phi)=h+\int_{0}^{360} w\left(\phi-\phi^{\prime}\right) H_{u_{0}}\left(u\left(\phi^{\prime}, t\right)\right) \mathrm{d} \phi^{\prime} .
$$

Three qualitatively different solution regimes are of interest for us:

(i) Ø-solution: $u(\phi) \leqslant 0, \forall \phi$.

Activation is negative over the whole field. It follows that $H_{u_{0}}(u(\phi)) \equiv 0$ and thus equation (7) reduces to

$$
u(\phi)=h .
$$

Since $u(\phi) \leqslant 0$, it follows that the condition $h \leqslant$ 0 is necessary and sufficient for the existence of the $\emptyset$-solution. Since this solution is required in order to be able to represent the absence of information (e.g., no target detected), we must restrict the valid domain of $h$ to be

$$
h<0 \text {. }
$$

As we will see next, the value of $h$ controls the existence of other equilibrium solutions.

3 An equilibrium solution, by definition, is a solution $u(\phi, t)=u(\phi)$, that is a solution which does not vary in time. Thus, $\dot{u}(\phi, t)=0$ for equilibrium solutions. 
(ii) $a$-solution: one connected region of excitation $R(u)=$ $\{\phi \mid u(\phi)>0\}$ of length $a$.

Here we exploit the fact that the system is translation invariant (by virtue of the assumed homogeneity of the field) and the pulse of width $a$ ( $a$-solution) can be centered on every value of the circular space. Under the assumption that the localized pulse is in the interval $[0, a]$, that is $u(\phi)>0$, for $\phi \in[0, a]$ and $u(\phi) \leqslant 0$ elsewhere, equation (7) reduces to

$$
u(\phi)=h+\int_{0}^{a} w\left(\phi-\phi^{\prime}\right) \mathrm{d} \phi^{\prime} .
$$

Let

$$
W(\phi)=\int_{0}^{\phi} w(\xi) \mathrm{d} \xi
$$

be the integral of $w(\Delta \phi)$. It is obvious that for a synaptic weight function of center-surround type $W(\phi)$ is an odd function (see figure 3 ) with only one maximum

$$
W_{\max }=\max _{\phi>0} W(\phi)=W\left(\phi_{0}\right)
$$

and

$$
W_{\min }=W(180)<0 .
$$

Since at the boundaries of the pulse the constraints $u(0)=u(a)=0$ hold, from equation (9), we get

$$
0=h+\int_{0}^{a} w(\xi) \mathrm{d} \xi=h+W(a)
$$

which reduces the question of the existence of a stationary pulse to the analysis of the roots of equation (12). As can be seen from figure 3 , for $-h<W_{\max }$ there exist two pulse solutions, one pulse of width $a_{1}$ and another pulse with width $a_{2}>a_{1}$.

(iii) $\mathrm{N}-b$ solution: $\mathrm{N}$ pulses of positive activation each with width $b$.

There are situations where we need to represent multivalued information, as for example when multiple targets, located at different locations, need to be represented. A neural field may, with an appropriate choice of parameters, exhibit equilibrium solutions consisting of multiple pulses which are separated by a minimal distance. We will now examine the conditions for the existence of these equilibrium solutions.

By virtue of the homogeneity of the field, we assume that the pulses may be centered at any value of the circular space, but with the constraint that the distance between any two pulses is sufficiently large ${ }^{4}$. The interaction strength between any two neurons $\phi$ and $\phi^{\prime}$ for sufficiently large distance $\left(>\phi_{s}\right)$ is constant and equal to $-w_{\text {inhib }}$ (see figure 2(a)).

We make the assumption that one pulse is located in the interval $[0, b]$ and that the remaining $\mathrm{N}-1$ pulses are located in the intervals $\left[\phi_{1}, \phi_{1}+b\right],\left[\phi_{2}, \phi_{2}+\right.$ $b], \ldots,\left[\phi_{N-1}, \phi_{N-1}+b\right]$, with $\phi_{i+1}-\phi_{i}>b+\phi_{s}, i=$ $1,2, \ldots, N-1$. That is, $u(\phi)>0$ for $\phi \in[0, b] \cup$ $\left[\phi_{1}, \phi_{1}+b\right] \cdots \cup\left[\phi_{N-1}, \phi_{N-1}+b\right]\left(\phi_{i+1}-\phi_{i}>b+\phi_{s}\right)$

4 This immediately imposes a limit on the number of pulses that can coexist.

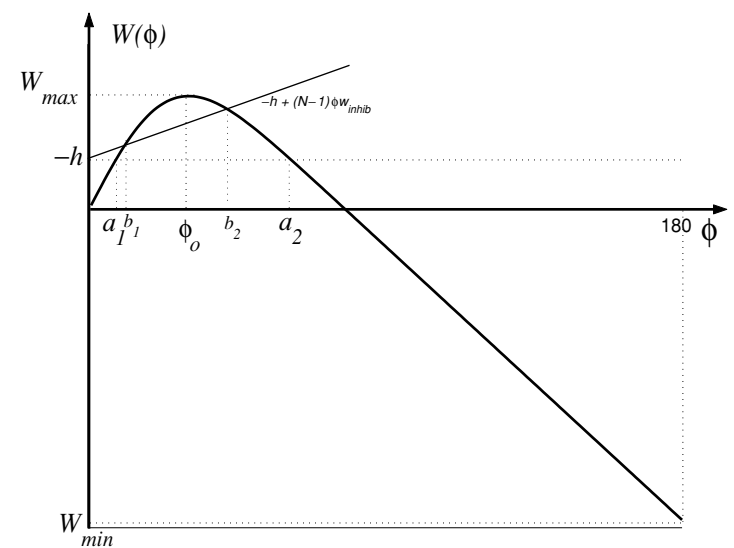

Figure 3. Integral of the weight function $w(\phi)$. Existence of equilibrium solutions: the intersection of the $-h$-level with the integral curve defines the width of the stationary $a$-pulse solutions. The $-h$-level intersects the integral curve at points $a_{1}$ and $a_{2}$. This means that two $a$-solutions exist, one $a$-solution representing a pulse of width $a_{1}$ ( $a_{1}$-solution) and second $a$-solution representing a pulse of length $a_{2}$ ( $a_{2}$-solution). The intersection of the integral curve with the line $-h+(\mathrm{N}-1) \phi w_{\text {inhib }}$ defines the existence of $\mathrm{N}-b$ solutions (see equation (14)). The field has two $\mathrm{N}-b$ solutions, one where the pulses have width $b_{1}\left(\mathrm{~N}-b_{1}\right.$ solution) and another where the pulses have width $b_{2}\left(\mathrm{~N}-b_{2}\right.$ solution). Stability of equilibrium solutions: the slope of the integral curve at the intersection points indicates the stability of the corresponding pulse solutions. The slope of $W(\phi)$ at $\phi=a_{1}$ is positive which indicates that the $a_{1}$-solution is unstable. Conversely, the negative slope of $W(\phi)$ at $\phi=a_{2}$ denotes that the $a_{2}$-solution is stable. $W(\phi)$ has positive slope at $\phi=b_{1}$ and negative slope at $\phi=b_{2}$. This reveals that the $\mathrm{N}-b_{1}$ solution is unstable while the $\mathrm{N}-b_{2}$ solution is stable.

and $u(\phi) \leqslant 0$ elsewhere. Equation (7) can then be written as

$$
\begin{gathered}
u(\phi)=h+\int_{0}^{b} w\left(\phi-\phi^{\prime}\right) \mathrm{d} \phi^{\prime}+\int_{\phi_{1}}^{\phi_{1}+b} w\left(\phi-\phi^{\prime}\right) \mathrm{d} \phi^{\prime} \\
+\cdots+\int_{\phi_{N-1}}^{\phi_{N-1}+b} w\left(\phi-\phi^{\prime}\right) \mathrm{d} \phi^{\prime} .
\end{gathered}
$$

Since at the boundaries of a pulse $u(0)=u(b)=0$ holds and

$$
\int_{\phi_{i}}^{\phi_{i}+b} w\left(\phi-\phi^{\prime}\right) \mathrm{d} \phi^{\prime}=-b w_{\text {inhib }},
$$

combining equations (13) and (10) we can conclude that the existence of a $\mathrm{N}-b$ equilibrium solution is determined by the roots of

$$
0=h+W(b)-(\mathrm{N}-1) b w_{\text {inhib }} .
$$

As can be seen from figure 3 , for $-h+\left((\mathrm{N}-1) b w_{\text {inhib }}\right)<$ $W_{\max }$ there exist two $\mathrm{N}-b$ pulse solutions: one where the pulses have width $b_{1}$ and another with pulses of width $b_{2}>b_{1}$.

It should be noted that if the interaction kernel $w(\Delta \phi)$ vanishes for large enough $\Delta \phi$, say $|\Delta \phi|>c$, then there can be many independent stable pulse solutions as long as their distance is at least $c$. 
2.1.2. Stability of equilibrium solutions. ${ }^{5}$ We now discuss the stability of the relevant solutions. The $\emptyset$-solution is always stable. For the discussion of the stability of a pulse solution, Amari developed a stability criterion and showed that a pulse of width $a$ is stable if

$$
\mathrm{d} W(a) / \mathrm{d} a<0 .
$$

Thus, from figure 3 we see that only the solution with equilibrium length $a_{2}>\phi_{0}$ is asymptotically stable and hence robust against perturbations. Likewise, a $\mathrm{N}-b$ solution is stable if

$$
\mathrm{d} W(b) / \mathrm{d} b<0 .
$$

Again, from figure 3 we see that the $\mathrm{N}-b$ solution with pulses of width $b_{1}$ is unstable while the $\mathrm{N}-b$ solution consisting of pulses of width $b_{2}>\phi_{0}$ is stable. In summary

1. The $\emptyset$-solution is always asymptotically stable and exists for $h<0$.

2. An $a$-solution is asymptotically stable if $\mathrm{d} W(a) / \mathrm{d} a<0$ and unstable if $\mathrm{d} W(a) / \mathrm{d} a>0$. An asymptotically stable $a$-solution exists for

$$
-W_{\max }<h<0 \text {. }
$$

3. A $\mathrm{N}-b$ pulse solution is asymptotically stable if $\mathrm{d} W(b) / \mathrm{d} b<0$ and unstable if $\mathrm{d} W(b) / \mathrm{d} b>0$. An asymptotically stable $\mathrm{N}-b$ solution exists for

$$
-W_{\max }+\left((\mathrm{N}-1) b w_{\text {inhib }}\right)<h<0 .
$$

For fixed $w_{\text {inhib }}$, the value of $h$ may thus be used to guarantee for a unique pulse solution and hence for the capacity to make a decision. The $a_{2}$-solution is bi-stable with the homogeneous rest state in which $u(\phi)<0$ for all $\phi$. This bi-stable behavior of the dynamic is crucial for implementing the memory function since a pulse does not emerge 'spontaneously' from rest state due to noise in the system. Only a sufficiently strong, localized input carrying information about a particular direction may switch the system into a persistent pulse solution. The memorized information may be updated, since the translation invariance of the system guarantees that new external input may displace the pulse to encode the newly arrived information (see below).

If for longer periods of time no sensory input is available, the working memory system should be switched back to resting level. For implementing this 'forgetting' mechanism in our robots, we exploit the fact that a pulse solution may be destabilized by adjusting the global inhibitory parameter $h$ to a value $\left|h_{\min }\right|>W_{\max }$. We use a first-order dynamics with an appropriate time scale for this adaptation process:

$$
\frac{\mathrm{d} h}{\mathrm{~d} t}=-r_{h, \min } c_{h}\left(h-h_{\min }\right)-r_{h, \max }\left(1-c_{h}\right)\left(h-h_{\max }\right) .
$$

Here, $\left|h_{\max }\right|<W_{\max }$ and $\left|h_{\min }\right|>W_{\max }$ are the two limit values of the resting level within the bi-stable and

\footnotetext{
5 Stable equilibrium solutions are patterns of activation that the neural field can maintain persistently, even in the presence of perturbations. It should be noted that 'stable' implies 'waveform stable' in the more strict sense.
}

the mono-stable regime, respectively. This dynamic lowers the resting level (destabilizing memory) at a rate $r_{h, \min }$ in the case that a memorized pulse exists $\left(c_{h}=1\right)$. It restores the maximal resting level (to enable memory) at the rate $r_{h \text {, max }}$ otherwise. The destabilization or forgetting process is slower than the restoring process, so that after forgetting, the field is immediately able to detect and memorize new information. The presence of a memorized pulse is represented by

$$
c_{h}=\left[H_{u_{0}}\left(N_{u}\right)-H_{u_{0}}\left(N_{s}\right)\right] H_{u_{0}}\left(N_{u}\right),
$$

where

$$
N_{u}=\int_{0}^{360} H_{u_{0}}(u(\phi)) \mathrm{d} \phi
$$

is the total positive activation in the field and

$$
N_{s}=\int_{0}^{360} S(\phi) \mathrm{d} \phi
$$

is the total external input activation (positive by construction). The function $c_{h}$ is equal to 1 if there is positive activation in the field $\left(N_{u}>0\right)$, but no input $\left(N_{s}=0\right)$. It is zero if there is no pulse in the field or if there is a pulse and also input.

2.1.3. The external input signal. The input or stimulus signal provides a means of coupling energy or initial excitation into the field. Since $S(\phi)$ is additively coupled in equation (2), we can easily see that when the input signal is very large compared to the nonlinear part of the field dynamics described by the integral term, the field behaves as a linear system with the asymptotically stable equilibrium solution $u(\phi) \approx S(\phi)+h$. For the applications, this behavior is not useful because the field only reproduces the input pattern. Thus, we have to guarantee that the external stimulus is weak compared to the integral term. Nevertheless, as discussed below, even a weak input signal, which is not sufficiently strong to drive the neuronal population beyond the activation threshold, $u_{\mathrm{TH}}$, for triggering a self-stabilized pulse, may have a major impact on the processing of subsequent stimuli.

When the initial state of the field is the flat solution (Øsolution), $u(\phi)=h<0 \forall \phi$, depending on the input strength the field can remain in the off-state or it can evolve a localized pulse. The question we have to address is the following: how much input energy is necessary to bring the system from the $\emptyset$-solution to a stable $a$-solution? For a mono-modal input, the critical stimulus energy that triggers this transition depends on the amplitude and on the spatial width of the input signal. To get an estimation of this critical energy, we consider a uniform input of amplitude $s^{\prime}$ and width $a^{\prime}$. The field activation converges to a stable $a$-solution if the following condition is satisfied:

$$
W(a)=-\left(h+s^{\prime}\right)
$$

The critical width $a\left(s^{\prime}\right)$, which depends on the amplitude $s^{\prime}$, can be found by inverting the function $W(a)$, i.e.,

$$
a\left(s^{\prime}\right)=W^{-1}\left(-\left(h+s^{\prime}\right)\right) \text {. }
$$

When the initial state of the field is a stable localized pulse with width $a_{0}$ centered at $\phi_{p}$, two situations may occur: 


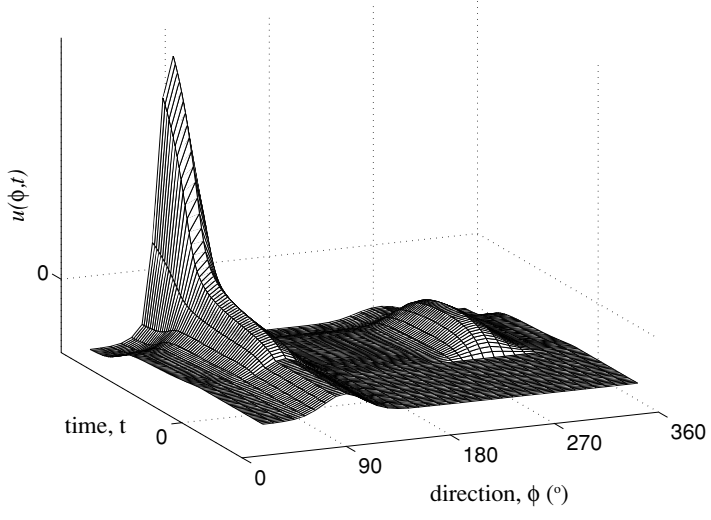

Figure 4. The evolution of a self-stabilized activity pattern representing the direction $\phi=90$ is shown. Time $t=0$ defines the onset of a bimodal input pattern. Note that at that time the neuronal population encoding $\phi=90$ appears to be already pre-activated.

The following parameters' setting was used (see section 2.3):

$\Delta \phi_{d}=\pi / 180 \mathrm{rad}$ (i.e. $2^{\circ}$ ), $\mathrm{d} t_{\text {Euler }}=0.06 \mathrm{~s}, \tau=5 \mathrm{~d} t_{\text {Euler }}$,

$h=-W_{\max } / 2, A=6, \sigma=15 \pi / 180, w_{\text {inhib }}=5$.

(i) The input signal is centered at $\phi_{\text {input }}$ and spatially overlaps with the interval $\left(\phi_{p}-a_{0} / 2, \phi_{p}+a_{0} / 2\right)$. In response to the external stimulus, the pulse moves in the direction of increasing input, searching for the maximum of $S(\phi)$. It stops when the inputs at the right and at the left boundary of the pulse are equal. At the same time, the width of the pulse changes slightly (for more details see Amari (1977)).

(ii) The input signal does not overlap with the self-stabilized activity pattern; then, to evolve a new pulse centered at field position $\phi_{\text {input }}$, the input at this site has to overcome not only the global inhibition level $h$ but also the additional inhibition $\left(-w_{\text {inhib }} a_{0}\right)$ created by the existing pulse of width $a_{0}$ at $\phi_{p}$. Thus, the input strength $S\left(\phi_{\text {input }}\right)$ must exceed the value $w_{\text {inhib }} a_{0}+h$, i.e., $S\left(\phi_{\text {input }}\right)>w_{\text {inhib }} a_{0}+h$.

In our robotic applications, if we need a field operating in the decision or selection mode then we are interested in the $\emptyset$-solution and $a$-solution only. Thus, we have to tune the parameters of the field to avoid the existence of $\mathrm{N}-b$ solutions. This is accomplished by making the inhibition strength $w_{\text {inhib }}$ sufficiently large such that condition (17) is satisfied but not condition (18). This way, the field exhibits a competition process among the various stimulated sites and eventually only one self-stabilized pulse evolves. The field converges to the stable $a$-solution in response to a multi-modal input. In the example of figure 4, a bimodal, completely symmetric input pattern is presented at time $t=0$. However, since the neuronal population encoding direction $\phi=90$ appears to be already pre-activated above resting level by a subthreshold input, the decision is biased towards that direction. Moreover, the preshaping mechanism strongly alters the time course of the decision variable (i.e., the build-up of the pulse). This in turn may influence whether or not the encoded information affects the integration-decision process in connected pools of neurons (Erlhagen et al 2006a).

If we need the field to represent and memorize the information represented by multiple inputs, then we have to

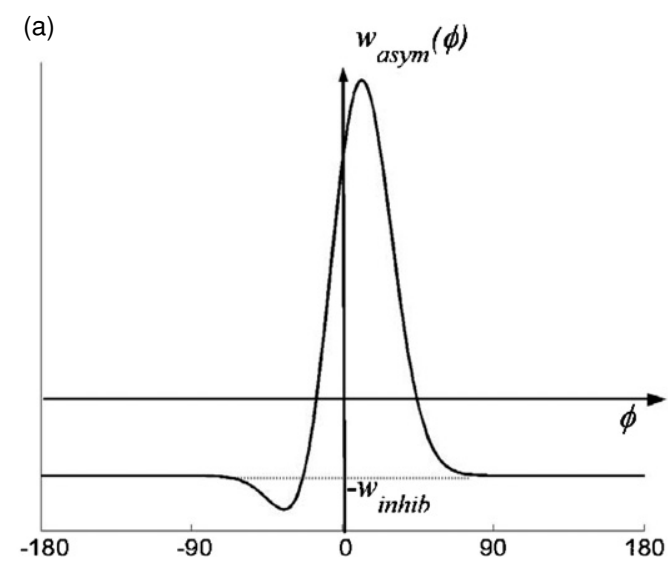

(b)

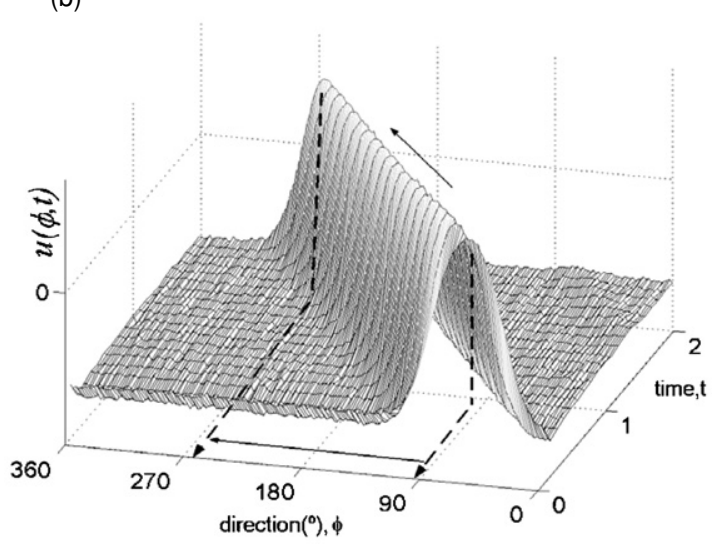

Figure 5. (a) Asymmetric weight function $w_{\text {asym }}(\phi)$. (b) Self-stabilized traveling wave in direction space.

tune the parameters to guarantee the existence of a stable $\mathrm{N}-b$ solution. In this case, a localized input signal that is sufficiently distant to any existing pulse may be memorized.

\subsection{Traveling pulse solutions}

A characteristic of HD cells is their ability to update their firing based on self-motion cues even in complete darkness. It is believed that when the head moves an angular head velocity signal from either vestibular or motor inputs shifts the localized activity pattern to new heading angles. The amount of displacement corresponds to the integral of the angular head velocity (Taube and Bassett 2003). Zhang (1996) proposed a dynamic modification of the lateral connections of the HD network to account for this shift mechanism. Let $w(\phi)$ be a symmetric synaptic weight function of center-surround type that supports a stable localized distribution $U(\phi)$ of the firing rates

$$
U(\phi)=h+\int_{0}^{360} w\left(\phi-\phi^{\prime}\right) f\left(U\left(\phi^{\prime}\right)\right) \mathrm{d} \phi^{\prime} .
$$

Zhang considers the weight function

$$
w_{\text {asym }}(\phi, t)=w(\phi)+\eta(t) w^{\prime}(\phi),
$$

where $w^{\prime}(\phi)$ is the derivative of the weight function $w$ and $\eta(t)$ the input that causes the head-direction shift (figure 5(a)). 
The full dynamic field equation then reads

$$
\begin{aligned}
& \tau \frac{\delta u(\phi, t)}{\delta t}=-u(\phi, t)+h \\
& \quad+\int_{0}^{360} w_{\text {asym }}\left(\phi-\phi^{\prime}, t\right) f\left(u\left(\phi^{\prime}, t\right)\right) \mathrm{d} \phi^{\prime} .
\end{aligned}
$$

An exact solution of this equation is given by

$$
u(\phi, t)=U\left(\phi+\frac{1}{\tau} \int_{0}^{t} \eta(s) \mathrm{d} s\right)
$$

which follows immediately from the observation that

$$
U^{\prime}(\phi)=\int_{0}^{360} w^{\prime}\left(\phi-\phi^{\prime}\right) f\left(U\left(\phi^{\prime}\right)\right) \mathrm{d} \phi^{\prime} .
$$

Thus, the weight function (26) may shift the activity profile without disturbing its shape (figure 5(b)), and the instantaneous angular velocity of this traveling activity wave is given by

$$
\varpi(t)=-\frac{\eta(t)}{\tau} .
$$

If the asymmetry within the network connections is proportional to the instantaneous angular speed of the head and the internal direction is initially aligned with the real head direction, the network works as a perfect velocity integrator. When also adding a component proportional to an acceleration term $(c>0)$

$$
\eta(t)=-\tau \varpi(t)-\tau c \dot{\varpi}(t),
$$

the peak of the traveling wave seems to lead the real head direction by a constant time $c$. Such an anticipatory firing pattern has indeed been described in HD cell populations (Taube and Bassett 2003).

Predictive representations associated with motion seem to be a widespread phenomenon in the cortex (Jellema and Perrett 2002, Eskandar and Assad 1999, Fogassi et al 2005; for a modeling account see also Erlhagen (2003)). They may be driven not only by motor output or sensory input but also by contextual cues. For the robotic applications, we use selfstabilized waves in position or direction space as an internal model for predicting the trajectory of the partner robot.

We end this section with the remark that there is a renewed interest by part of the mathematics community to analyze pattern formation in dynamic neural fields. The analytical results given by Amari have been generalized to the case of two spatial dimensions (e.g., Taylor (1999), Laing and Troy (2003)). From the robotics point of view these results are interesting since they allow storing in a single representation for instance the direction and distance of objects. Moreover, new insights have been gained about the existence and shape of localized pulse solutions when linear interactions are combined with bi-stable field elements (Horta and Erlhagen 2006). The results reveal a dependence of the pulse solutions on parameters such as input strength and width which may also be exploited for the robotic applications.

\subsection{Discretization and numerical computation}

The activation $u(\phi, t)$ depends not only on time $t$, but also on the continuous variable $\phi$. For the applications, the continuous field is evaluated at discrete points in space and time. The field dynamic is integrated numerically using the forward Euler method. Let $\mathrm{d} t_{\text {Euler }}$ be the Euler time step and $\Delta \phi_{d}$ the sampling distance along $\phi$. The field activation, the external input and the weight function are then vectors that can be indexed:

$$
\begin{gathered}
\phi_{j} \doteq \phi\left(j \Delta \phi_{d}\right), \\
u_{j, i} \doteq u\left(j \Delta \phi_{d}, i \mathrm{~d} t_{\text {Euler }}\right), \\
S_{j, i} \doteq S\left(j \Delta \phi_{d}, i \mathrm{~d} t_{\text {Euler }}\right), \\
w_{j} \doteq w\left(j \Delta \phi_{d}\right),
\end{gathered}
$$

where $j\left(=0,1,2, \ldots, N_{j}\right)$ is the index of the spatial dimension, $\phi$, and $i(=0,1,2, \ldots)$ is the index of the discrete time. With these conventions and applying the forward Euler method to equation (2), the integration of the homogeneous neural field reads

$$
\begin{array}{r}
u_{j, i+1}=u_{j, i}+\frac{\mathrm{d} t_{\text {Euler }}}{\tau}\left[-u_{j, i}+S_{j, i}\right. \\
\left.+\sum_{k=0}^{N_{j}} w_{j-k} f\left(u_{k, i}\right) \Delta \phi_{d}+h\right] .
\end{array}
$$

To guarantee numerical stability, the following condition must hold:

$$
\mathrm{d} t_{\text {Euler }} \ll \tau \text {. }
$$

The Euler time step is the smallest time unit that exists in the overall dynamical system. It is equal to the computational cycle time of the computer running the numerical integration. Its value imposes minimal time scales on the entire dynamic architecture. Discretization in the space dimension can produce behavior qualitatively different from the continuous case. Fortunately, however, if the sampling distance along $\phi, \Delta \phi_{d}$, is chosen to be sufficiently small, the behavior in the discrete case approximates the behavior of the continuous field equation quite well.

\section{Application of dynamic neural fields in joint action tasks}

\subsection{Joint search task}

As a first example for illustrating how we apply dynamic neural fields to implement some cognitive capabilities in autonomous robots, we have chosen a joint search task. A team of two mobile robots has to find objects distributed in the workspace and transport them to a predefined area. We assume that there is no direct communication and the motor intention of the partner has to be inferred from observed movements.

Since multiple objects may be simultaneously sensed, each robot should be able to make a decision about the object to be attended next. To guarantee an efficient team strategy, this decision process should reflect an adequate division of the search space between the two agents. This necessarily requires 
the ability to predict future positions of the partner based on past trajectory information and additional contextual cues. Moreover, since 'occlusion is one of the most fundamental facts about vision in natural scenes' (Shimojo and Nakayama 1990), a continuous stream of sensory information about the attended object and the moving partner is not guaranteed. This lack of 'bottom-up' information during brief periods of time should of course not disrupt the ability to organize goaldirected behavior.

3.1.1. Neuro-inspired dynamic field architecture. In the following, we summarize experimental findings, which constrain our choice of the dynamic control architecture and the nature of the internal representations used to model joint action.

The prefrontal cortex (PFC) has long been suspected to play a central role in cognitive control by establishing the proper mappings between sensory inputs, internal states (e.g., stored memories, predictions) and motor outputs needed to perform a given task (for a review see Miller (2000)). PFC is an interconnected set of cortical areas which is anatomically linked to virtually all sensory and motor systems. It is thus well positioned to integrate diverse information and subsequently bias the processing in other brain structures towards the achievement of an intentional goal. Indeed, the main behavioral effect of prefrontal cortex damage seems to be the incapacity to override more salient or habitual responses and thus to persist towards a goal (Miller 2000). For the present joint search task, the goal for each robot is to select an object for placing it in the target area taking into account the behavior of the other robot. Consequently, the goal representation in PFC should organize sensory-motor mappings that go beyond the habitual decision to move towards the most salient, that is, closest object.

The fact that many PFC neurons sustain their activity over extended periods of time has been interpreted as evidence that these neurons actively maintain goal-relevant information. Importantly, specific information about for instance object location or object identity seems to be stored in distinct subpopulations. The working memory capacity of PFC ensures that potential target objects in joint action tasks may affect the behavior even in the presence of interfering events such as occlusion which disrupt the spatiotemporal continuity of sensory input.

The cognitive capacity to infer the action goal of conspecifics is important enough, for all species living in social groups, that specific brain mechanisms have evolved. Neurophysiological studies in humans and monkeys reveal that the cortex in and near the superior temporal sulcus (STS) region is involved in analysis of biological motion (Jellema and Perrett 2002). The STS region is activated by the observation of eye, head, hand or body movements. Important for the present study is the finding that these high-level visual representations may include sensitivity to intentionality. Perrett and colleagues (Jellema and Perrett 2002, Baker et al 2001) described a population of neurons in area STS of macaque monkey that increased their firing rate when the experimenter disappeared behind a screen. Furthermore, this

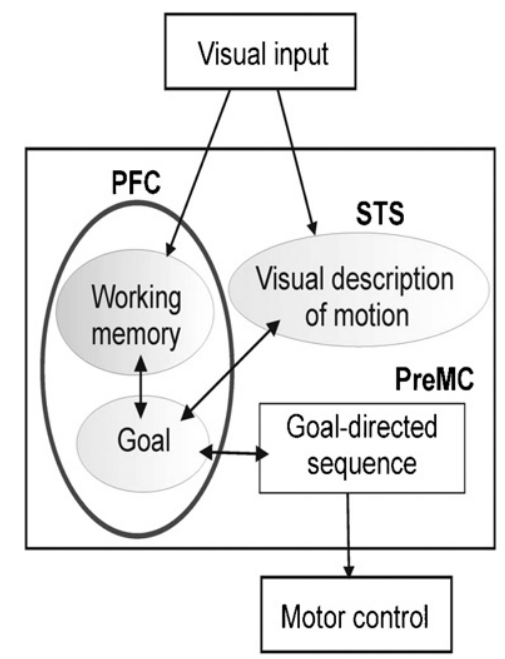

Figure 6. Schematic view of the architecture for the joint search task. Layer STS represents the motion of the partner robot in form of a self-stabilized activity wave in a neuronal population encoding direction. The working memory (WM) layer in PFC contains a population which memorizes the visual input about the location of the objects. The inputs from STS (inhibitory) and WM (excitatory) lead to a decision in form of a self-stabilized activity pattern representing the goal object which the robot will attend next. The goal representation is linked with populations in premotor areas encoding an action sequence (approaching-reaching-grasping) which has to be performed to achieve that goal.

increase was selective for the position of occlusion within the test room. The authors conclude from their analysis of the firing pattern that it is consistent with the idea that these populations "code not only for the presence of the experimenter behind the screen, but also for the intention of the experimenter to go behind that screen' (Jellema and Perrett 2002). We have recently suggested that a self-stabilized traveling wave in a neuronal population encoding position may underlie this form of motion extrapolation (Erlhagen 2003). Being able to track a partner even in the presence of occluding surfaces and to predict future positions is highly advantageous in joint action tasks since it allows the observer to quickly adjust his/her actions accordingly.

In figure 6 we summarize the robot control architecture for the joint search task which conceptualizes the described neurophysiological findings. The working memory (WM) layer of PFC stores the sensory information about the location of objects. The goal layer in PFC gets input from working memory and from layer STS which encodes the predictive information about the partner's position in the immediate and distal future. Finally, it is assumed that the goal representation projects to motor areas (most likely premotor cortex (PreMC)) in which an action sequence such as approaching-reachinggrasping necessary to achieve that goal is represented (Miller 2000).

All layers of the STS-PFC circuit are formalized by dynamic neural fields spanned over the space of direction relative to an arbitrary but fixed frame of reference. In the goal layer, the recurrent interactions allow for just one localized activity pattern whereas in the WM layer several pulses may 


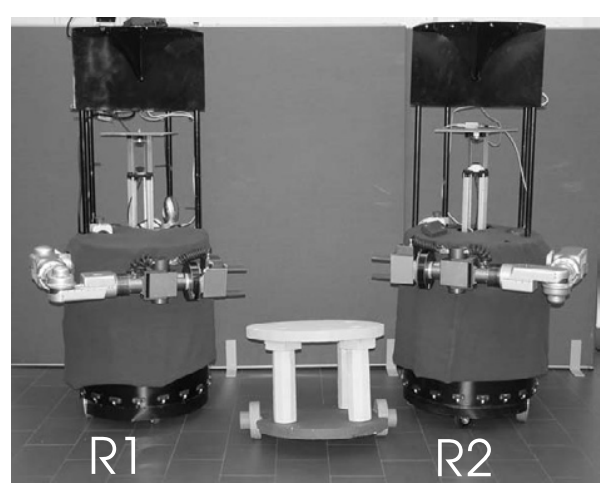

Figure 7. The two mobile robotic platforms are shown which are equipped with a 7-degrees-of-freedom arm/gripper, infrared sensors, an omnidirectional and a monocular camera system.

coexist since the interaction kernel does not extend over the whole field. The dynamic mode for the STS layer is an anticipatory wave. It travels with a velocity larger than the directional change per time unit of the partner robot which is estimated initially from direct sensory input. The dynamics in the goal field evolves under the influence of the excitatory input $S_{\mathrm{WM}}$ from the WM field and the input $S_{\mathrm{STS}}$ from the STS field which is assumed to be inhibitory:

$$
\begin{gathered}
\tau \frac{\delta u_{\mathrm{G}}(\phi, t)}{\delta t}=-u_{\mathrm{G}}(\phi, t)+h-S_{\mathrm{STS}}(\phi, t)+S_{\mathrm{WM}}(\phi, t) \\
\quad+\int_{0}^{360} w\left(\phi-\phi^{\prime}\right) f\left(u_{\mathrm{G}}\left(\phi^{\prime}, t\right)\right) \mathrm{d} \phi^{\prime} .
\end{gathered}
$$

The read-out of the firing pattern in the input layers is made in every time step by applying the ramp nonlinearity (6) to the normalized activation $u_{\mathrm{STS}} /\left|u_{\mathrm{STS}}\right|_{\infty}$ and $u_{\mathrm{WM}} /\left|u_{\mathrm{WM}}\right|_{\infty}$, respectively.

3.1.2. Hardware requirements and implementation issues. Although the focus of this tutorial is on the design principles of the dynamic field architecture, we briefly overview the sensory processing and path planning which we use to date for the experimental validations on the real robot platforms shown in figure 7. Each robot has an omnidirectional vision system which allows tracking the partner robot based on form and color information. It also provides the dynamic field model with an estimate of the object locations. We use an additional monocular camera system mounted on the torus to get a more precise information about object position and orientation for the grasping. Videos of the robots in action can be found at http://www.dei.uminho.pt/pessoas/estela.

Once a decision about the next object to be attended is made, the mobile platform first has to move towards that target thereby avoiding obstacles. Information about obstacles is provided by infrared sensors mounted on a ring which is centered on the robot's rotation axis. To generate overt behavior, we follow the principles of the dynamic approach to behavior-based robotics (Schöner et al 1995, Bicho et al 2000). The state variable of the dynamical system controlling the robot is the heading direction. The vector field is shaped by an attractive force representing the desired target direction and repulsive forces representing the direction of obstacles which have to be avoided. The rate of change of heading direction obtained from the dynamics is directly used to control the rotation speed of both wheels (for details see Bicho (2000)). The dynamic approach could be, in principle, used to control also the reaching and grasping of the 7-degrees-of-freedom arm/gripper (AMTEC, Germany). Since we have yet not properly explored this possibility (but see Iossifidis and Schöner (2004)), we employ instead a global planning method in posture space which is inspired by Mel's biologically plausible network model (Mel 1990; for details see Erlhagen et al (2006b)).

3.1.3. Robot experiments. In figure 8 we show the dynamics of the field model and the simulated behavior of the robots in a situation which is completely symmetric for both robots, $\mathrm{R} 1$ and R2. They sense two objects, T1 and T2, which are located to the left and the right of the target areas (gray circles) where the two objects have to be placed. Robot R1 is initially heading towards object T2 (time $t_{0}$ ). However, as shown by the anticipatory wave in layer STS, R1 predicts that T2 is also the current action goal of R2. The inhibitory input from STS starts to decrease the localized activity pattern in the goal layer representing T2 (time $t_{1}$ ). Finally, the summed input from STS and WM results in a switch of the goal representation to T1 (time $t_{2}$ ) and robot R1 starts to direct its behavior towards that object. This occurs despite the fact that the sensory input from $\mathrm{T} 2$ is stronger since the object is closer.

Note that normally the timing of decisions is different in each agent. It is thus unlikely that also robot R2 will change its initial motor intention based on the predicted outcome of the partner's action.

The second example shall illustrate the importance of working memory in joint search tasks (figure 9). Both robots are again heading towards the same object T2 (time $t_{0}$ ). Robot $\mathrm{R} 1$ has initially sensed and memorized also the direction of object T1. However, this object disappears from sight during the move due to an occluding surface (time $t_{1}$ ). The selfstabilized wave in layer STS again indicates the conflict with the motor intention of robot R2. Since no direct sensory input is available, the switch to goal T1 occurs now completely based on memorized information (time $t_{2}$ ). When sensory information is again available, the directional information about T1 is updated (time $t_{3}$ ).

Figure 10 illustrates that the self-stabilized wave in layer STS may link the ongoing behavior of the partner robot to a particular object even when most of the motion trajectory is hidden from view. Robot R2 disappears behind a wall but the initial information about its change in direction is sufficient to trigger the predictive signal consistent with the hypothesis that $\mathrm{R} 2$ is heading towards $\mathrm{T} 1$. As a consequence, robot R1 directs its attention towards object $\mathrm{T} 2$ since a whole range of heading directions including the direction of T1 appears to be inhibited.

\subsection{Action understanding in a grasping-placing task}

Although the experiments in the joint search task show that the capacity to extrapolate trajectory information results in an 

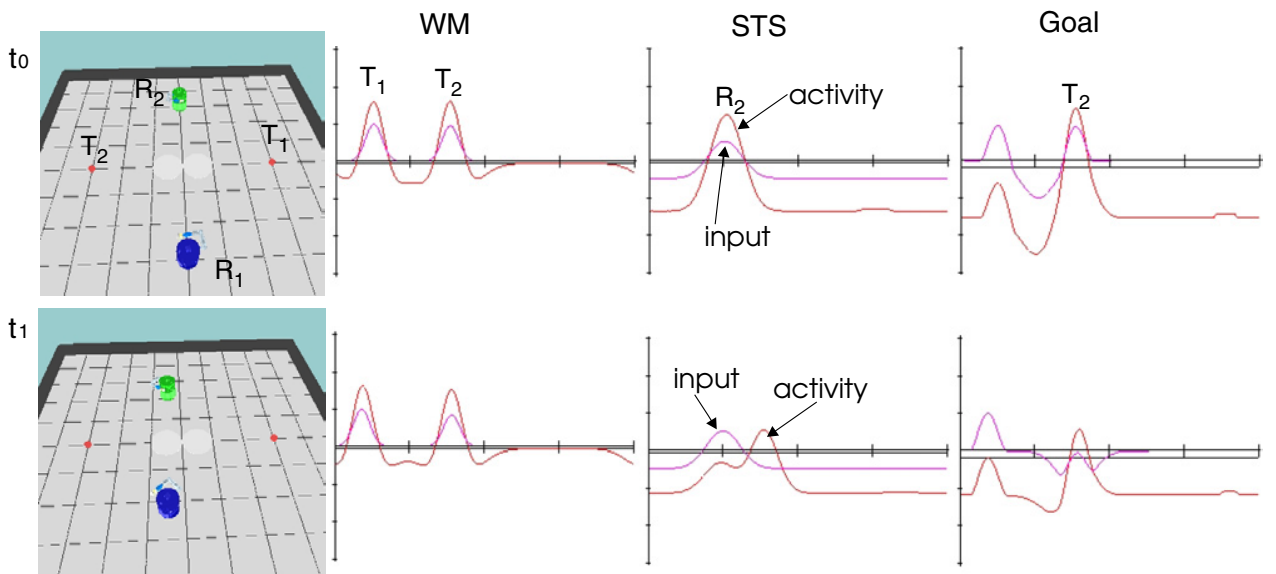

t2
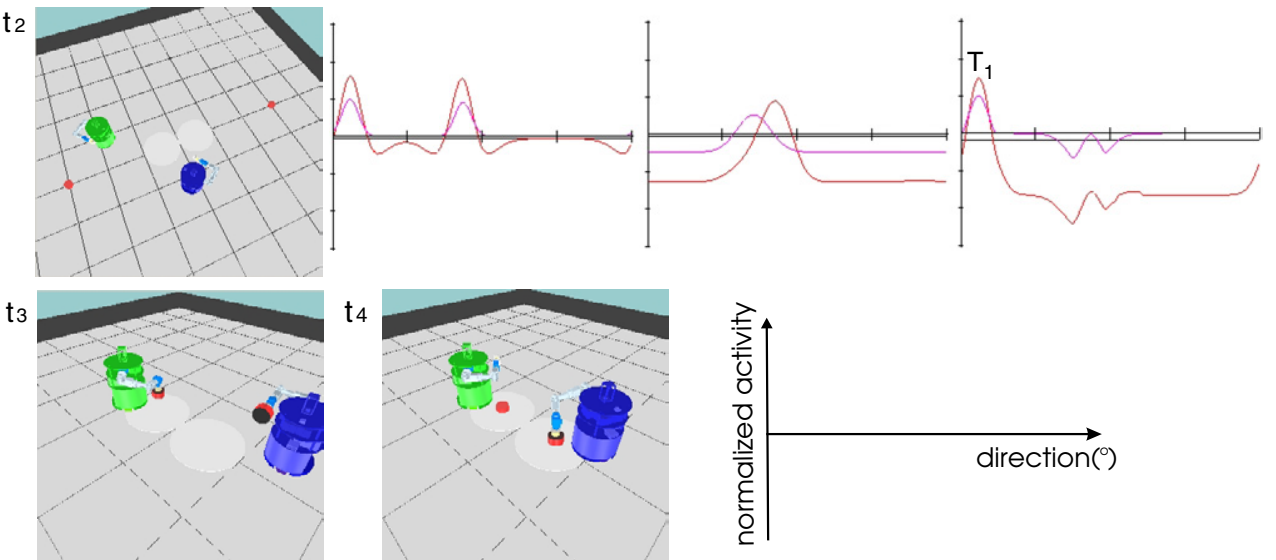

$\mathrm{t}_{4}$
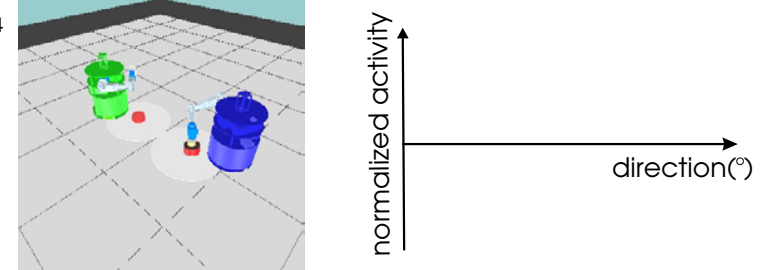

Figure 8. Snapshots at different points in time of the dynamic model controlling robot R1 show the activity (thick line) of the three dynamic fields STS, WM and goal together with the input (thin line). The two localized peaks of activation in WM represent the location of the targets (T1 and T2). At time $t_{0}$, the decision to head towards target T2 is represented in the goal field. The other robot, R2, also moves towards T2. In STS, the peak of activation, representing the location of the partner, moves ahead of the input thus representing an anticipatory wave. As can be seen in the snapshots at times $t_{1}$ and $t_{2}$, the pulse in STS travels in the direction of T2. The inhibitory input from STS to the goal layer eventually inhibits the decision to move towards T2 and the robot switches its action goal, i.e., the decision is now to grasp object T1 (snapshot $t_{2}$ to $t_{3}$ ), while the partner keeps moving towards T2.

efficient division of the search space between the two agents, the wave mechanism alone is not sufficient to efficiently organize more complex joint action tasks. Imagine, for instance, that there exist several objects in the current heading direction of R2. The observing robot R1 has to infer which of the objects is the current goal of the partner since the predicted outcome will affect the choice of its complementary action. This prediction may be possible by integrating additional information sources such as for instance object cues (e.g., color, shape) or memorized task information. Moreover, very often more detailed information about the action means (e.g., the grip type) may allow for inferring what the partner is going to do with a particular object.

In the following, we describe and validate a dynamic field model for action understanding which we have developed in close cooperation with our experimental partners (Erlhagen et al 2006a).

3.2.1. Extended field model. Converging lines of experimental evidence suggest that the interpretation of others' actions is not based on purely visual mechanism but involves also the observer's motor system. It has been suggested that an understanding of an observed movement as directed towards a specific goal may be gained by simulating the action outcome using the proper motor repertoire (Gallese and Goldman 1998). Compelling neurophysiological evidence for such a matching of action observation and action execution came from the discovery of the STS-PF-F5 mirror circuit (for a recent review see Rizzolatti et al (2001)). Mirror neurons first described in the premotor cortex (F5) of the macaque monkey fire both when the monkey performs a particular goal-directed action (e.g., grasping an object) and when it observes another individual performing a similar action. In the visual motion area STS, neurons representing actions similar to those coded by F5 have been described. The only difference seems to be that STS neurons do not fire during active movements. Both areas are linked to area PF of the parietal cortex which also contains mirror neurons. Recent neurophysiological findings in this area give direct support for the involvement of the mirror circuit in intention reading. Fogassi et al (2005) described a population of grasping mirror neurons which showed a selective response in dependence on the final goal of an action 


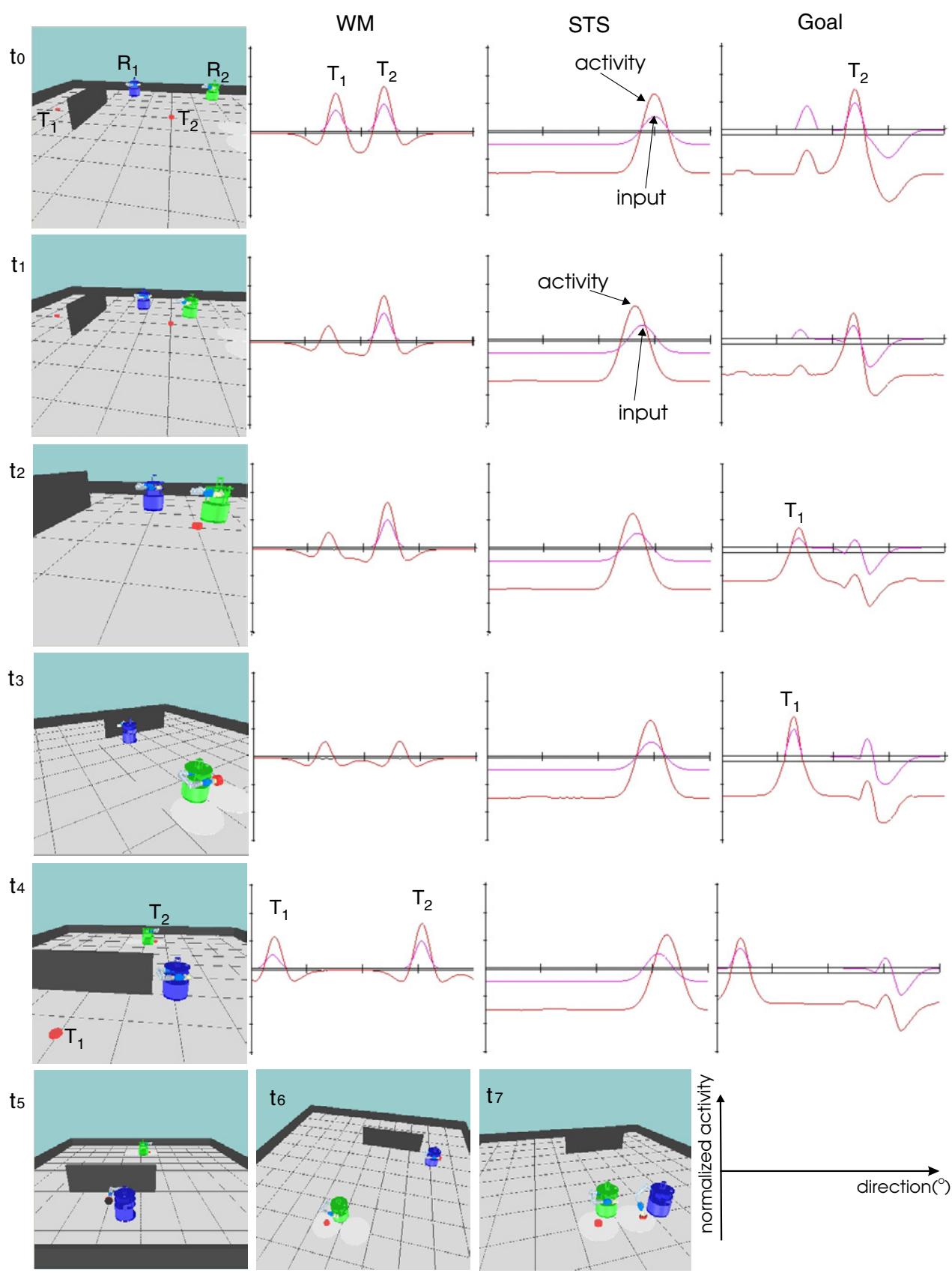

Figure 9. The importance of working memory for the joint search task is illustrated. Initially (snapshot $t_{0}$ ), both robots are moving towards object T2. R1 sees both objects and their location is represented in WM. At time $t_{1}$, object T1 is no longer visible since it is occluded by the wall. Its position remains represented in WM by a self-stabilized pulse (albeit with somewhat decreased amplitude). The traveling pulse in STS representing the motion of the partner ends up inhibiting the decision to move towards object T2 (time $t_{1}$ to $t_{2}$ ) which causes a switch in the decision. R1 moves now towards the occluded object T1 (snapshots $t_{2}$ to $t_{5}$ ) while R2 maintains the decision to grasp object T2.

sequence (grasping for eating versus grasping for placing). Since PF has strong anatomical connections to the prefrontal cortex, the PFC-mirror circuitry sketched in figure 11(b) may constitute a distributed neural network in which the meaning of an action is constructed based on working memory, sensory evidence, contextual cues and a goal-directed matching of action observation and action execution.
3.2.2. Experimental paradigm. To test the functionality of the field model in robots, we have chosen an action observation task in which a human model first grasps and subsequently places an object at one of two laterally presented targets that differ in height. The grasping and transporting behavior is constrained by an obstacle in form of a bridge (figure 11(a)). Depending on the height of the bridge, the lower target may 


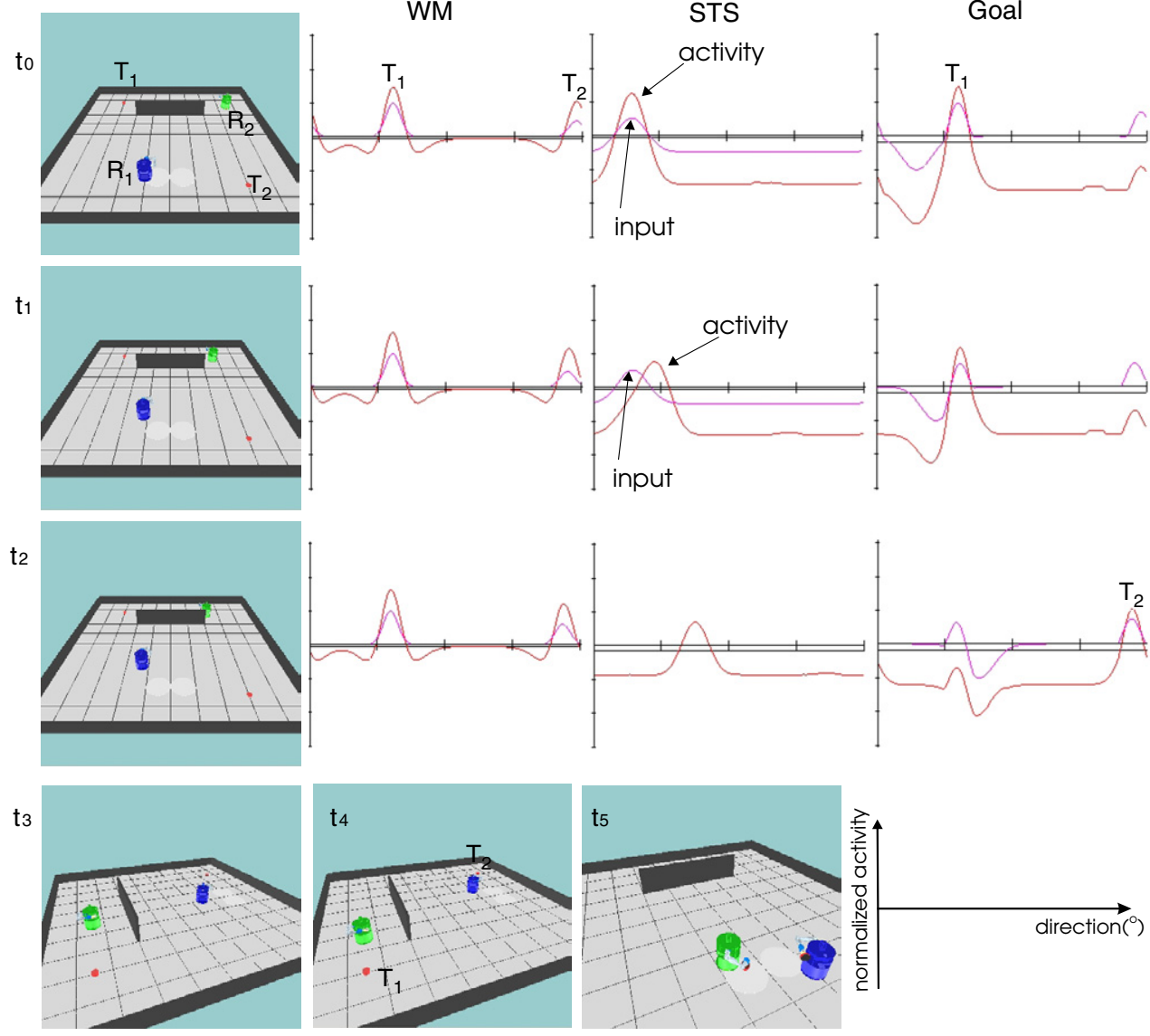

Figure 10. The impact of the capacity to track motion also behind occluding surfaces is illustrated. As represented in the goal layer, initially robot R1 heads towards target T1. In the time interval $t_{1}$ to $t_{2}$, robot R2, which is also heading towards T1, disappears behind the wall. The self-stabilized wave in STS representing the motion of the partner eventually inhibits the decision to grasp object T1 and robot R1 switches to object T2. This decision is represented at time $t_{2}$ in the goal field by an activation peak centered at the direction in which T2 lies.

(a)

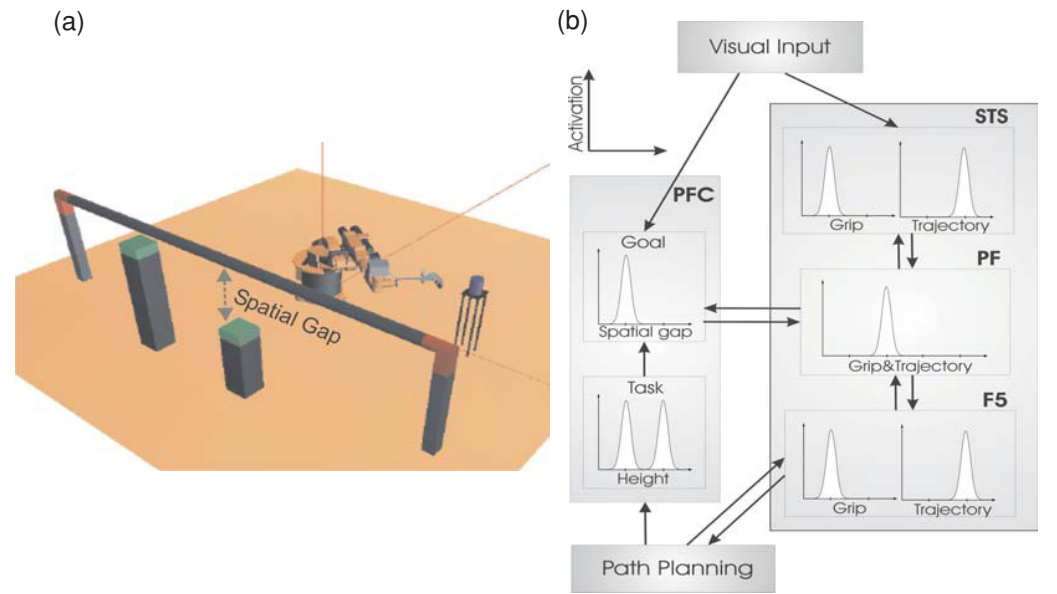

Figure 11. (a) Bridge paradigm: the robot has to grasp an object and place it at one of the two targets behind the bridge obstacle.

(b) Sketch of the control architecture. The central part consists of the STS-PF-F5 mirror circuit for a matching of action observation and action execution. The circuit is connected with the goal layer in prefrontal cortex (PFC) which encodes the intentional action goal (placing target) parameterized by its spatial gap relative to the bridge. The demonstrated means in STS, the selected goal, the selected action sequence in PF and the selected motor primitives in the action layer F5 are represented by self-stabilized activity patterns of local pools of neurons. The bimodal activity distribution in the task layer of PFC reflects the memorized information about the two (equally likely) placing goals parameterized by their height. 


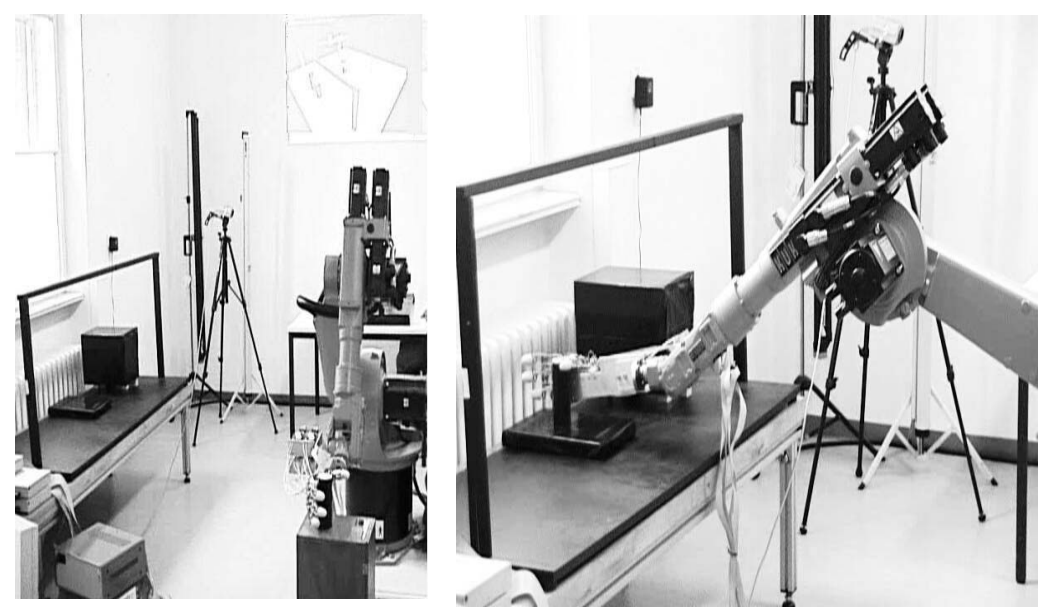

Figure 12. The robot in the bridge paradigm is shown. It consists of an industrial 6-degrees-of-freedom manipulator with a four-fingered anthropomorphic hand and a vision system.

only be reached by grasping the object with a full grip and transporting it below the bridge. Placing the object at the higher target, on the other hand, may require combining a precision grip and a hand trajectory above the bridge.

The fundamental idea for the processing in the STS-PFF5 circuit is that the matching takes place on the level of motor primitives which represent whole goal-directed motor behaviors and abstract from the fine details of the movements. For the bridge paradigm, we distinguish between two grasping primitives (precision grip (PG) and full grip (FG)) and two types of transporting primitives for avoiding the obstacle (below (BT) or above (AT) the bridge). The vision system classifies the demonstrated action in terms of grip type and trajectory type and triggers the respective representations in the visual layer STS. It is assumed that these primitives are already in the motor repertoire of the observing robot, that is, corresponding representations exist in the motor layer F5. In the intermediate layer PF, specific combinations of primitives (e.g., PG/AT) are stored which allow achieving a particular placing goal. The internal goal representations in PFC are parameterized by their height relative to the bridge (spatial gap in figure 11(a)). They may be triggered or influenced by direct visual input (placed object), through the connections to layer PF, learned associations with representations of object cues (e.g., color) and/or memorized task information (e.g., number and height of goals).

For each model layer, we use a dynamic neural field of center-surround type (1) and a sigmoid output function (5). Since the underlying metrics for abstract dimensions such as grip type are not known, it is further assumed that pools of neurons representing alternative action means (e.g., full or precision grip) interact only through inhibitory surround connections, thus implementing a competition process.

3.2.3. Robot platform and implementation issues. First experiments within the bridge paradigm were conducted on a robot platform consisting of an industrial 6-degreesof-freedom robot arm (KUKA, Germany) on which a four-fingered anthropomorphic robot hand (GRAALTECH, University of Genova, Italy) was mounted (see figure 12).

Videos of the robot in action can be found at http://www.dei.uminho.pt/pessoas/estela. For the imitation task, the vision system has to classify the hand motion of the human demonstrator. The hand and the object are identified and tracked in real time on the basis of a chroma-space blob segmentation in the YUV color space using a monocular camera view. The hand tracking algorithm is based on a mutual information optimization approach (Viola and Wells 1995) which maximizes the consistency between an observed image and postures of a hypothetic hand model. The hand trajectory (above or below the bridge) and the placing goal (high or low) are classified on the basis of a distance measure relative to the respective object. The classification of the grasping behavior (full or precision) is essentially based on the orientation of the palm relative to the object.

3.2.4. Robot experiments. The STS-PF-F5 mirror circuit implements a direct mapping from action observation onto action execution. To give a concrete example of the dynamics within the mirror circuit, imagine that in the action observation layer STS, the grip PG and the trajectory AT are represented. The two pulses act as additive input to the neural population encoding the sequence PG/AT in layer PF. The evolving pulse solution in PF triggers in turn through congruent mappings the respective neural representations, $\mathrm{PG}$ and $\mathrm{AT}$, in the action layer F5. As shown in our previous work, this pathway may be exploited to copy action means from the human demonstrator. However, due to differences in embodiment and/or environmental constraints, very often this matching cannot be direct. Imagine for instance that the bridge obstacle is lowered for the human but not for the robot. Now the human may grasp the object with a full grip (FG) to place it at the higher target. For the imitator, an automatic copying of the grip would lead to a collision with the bridge. Therefore, some cognitive control over the direct pathway is required. We exploit here a purely temporal mechanism to guarantee that the previously established pathway linking the PG/AT 
representation to the goal representation in PFC dominates the processing in the mirror circuit. Since in a known task setting the pulse in PFC evolves faster compared to the pulses in STS, the goal pathway biases the competition process between the two PF populations encoding the sequence PG/AT and FG/AT, respectively (Erlhagen et al, 2006a).

The following goal inference task shall illustrate how a simulation based on motor representations may be used to predict the sensory consequences of an ongoing action (Gallese and Goldman 1998). Recently, Umiltà et al (2001) have shown that a population of mirror neurons in F5 may encode a goaldirected action also when the crucial part defining that action is hidden from view. The information sufficient to trigger grasping mirror neurons was a hand disappearing behind an occluding surface combined with the knowledge that there is also a graspable object behind that occluder.

Adapted to the bridge paradigm this means that only the grasping part of the action sequence can be observed, the placing is hidden from view (figure 13(a)). The additional input necessary for goal inference comes from the information about potential end states represented as two self-stabilized pulses in the working memory layer of PFC. This task information acts as subthreshold input resulting in a preactivation close to threshold, $u_{\mathrm{TH}}$, of neuronal populations representing goals (in PFC) and associated sequences of means (in PF). As a consequence of the robot's 'expectation' prior to action observation, the activity pattern representing the observed grip type (FG) in layer STS is sufficient to trigger first the evolution of a pulse in $\mathrm{PF}$ representing the motor sequence FG/BT and subsequently the associated representation of the lower placing goal in PFC. The stable state of the model dynamics (figure 13(b)) reveals that this occurs despite the lack of a suprathreshold representation for the trajectory type in layer STS. Without pre-activation, the converging input from both STS inputs would be necessary to drive the population in layer PF. As depicted in figure 13(c), the robot shows its understanding by combining a full grip (FG) and a trajectory below the bridge (BT) to reproduce the inferred action effect. To generate the overt behavior, the abstract primitives represented in layer F5 are translated into the right kinematics using a global path planning in posture space (for details see Erlhagen et al (2006b)).

The experiment highlights the important role of common task knowledge for joint action. This task knowledge should be continuously updated (e.g., number and probability of placing goals) in accordance with the outcome of each individual imitation trial (see Erlhagen and Schöner (2002) for a computational implementation).

Movement primitives do not encode the fine details of the movements and thus provide a sufficiently abstract level of description for a matching across dissimilar embodiments. However, a remaining problem for goal inference in joint human-robot tasks is the large difference in motor skills. Very often, a direct matching through congruent motor primitives will be impossible. The robot may nevertheless acquire a meaning of an observed movement not strictly in its motor repertoire by covertly using its own action means for achieving the observed end state. Neurophysiological evidence for such (a)

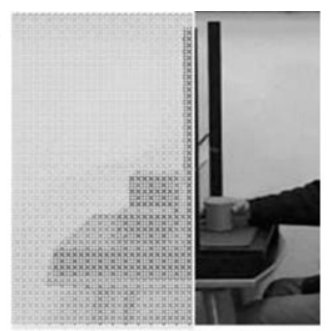

(b)
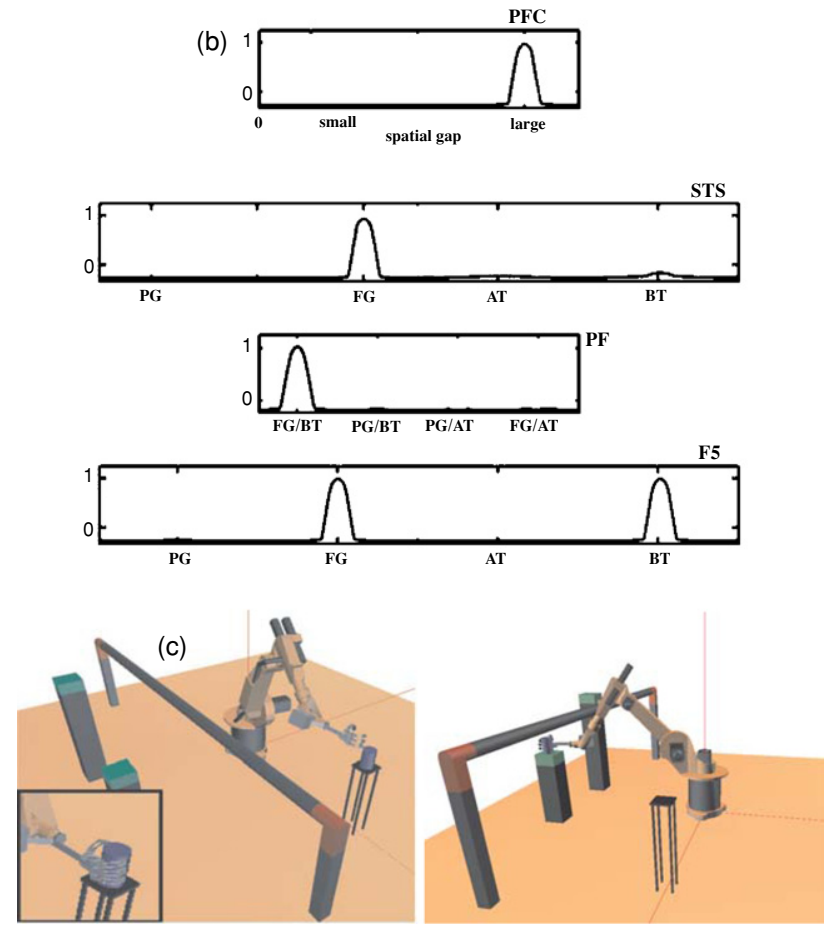

Figure 13. Goal inference task. (a) Only the grasping behavior of the human model is observable. (b) The stable pulse in layer PFC of the field model represents the inferred goal (lower placing target). Note that no visual description of the trajectory type is represented in layer STS. (c) To reproduce the inferred end state, the robot combines a full grip (FG) followed by a trajectory below the bridge (BT) as represented in the action layer F5 of the field model.

a learning mechanism comes from a recent study by Ferrari et al (2005) in which grasping neurons in F5 were described which fired when the monkey observed actions performed with an unknown tool. Importantly, the monkey could gain during practice an understanding of the movement as goal directed since the tool was used from time to time to give food.

The next experiment illustrates in a simulation the learning process for the robot (figure 14). The validation on the real robot is part of our future work. The human model grasps the object with a tool and places it at the higher goal. The vision system is assumed to classify this hand-tool motion as the new grip type 'IG' which differs from a full or precision grip (see Rittscher et al (2003) for a recent overview about different tracking and classification techniques). Although the tool use is not in its motor repertoire, the robot learns to associate the IG grip with the PG/AT sequence previously used to achieve the goal. Since the goal is known to the robot, the observation 
(a)

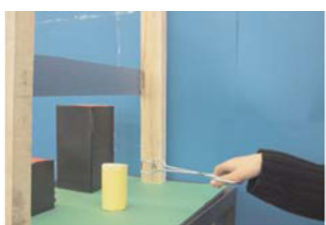

(b)
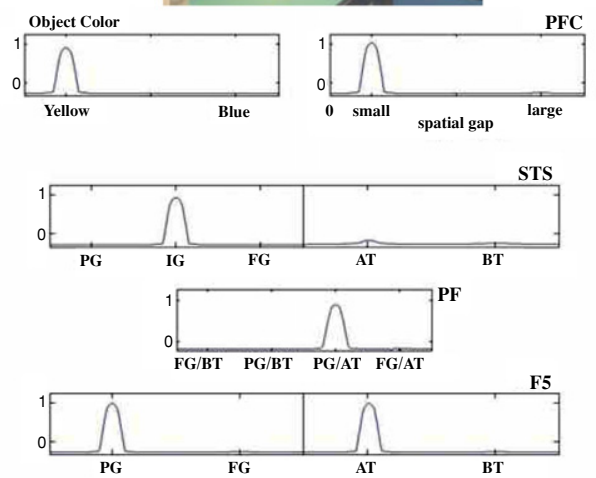

(c)

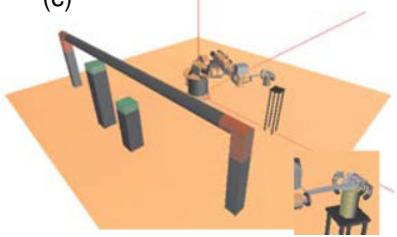

Figure 14. Learning to understand the meaning of an action (tool use) which is not in the motor repertoire of the robot. (a) The human model uses a tool to grasp the object. (b) The figure shows the stable state of the model dynamics in a goal inference task after the learning of synaptic links between the visual description of the new grip type IT in layer STS and the sequence PG/AT in layer PF. The robot usually uses this sequence to place the object at the higher target. Although the robot cannot reproduce the tool use, it can achieve an understanding of its meaning by simulating the goal using its own motor repertoire. For more details see the text. (c) A simulation of the overt behavior in the goal inference task is shown.

of the placing drives the goal representation in PFC and the associated sequence of motor primitives in PF. Subsequently, synaptic links between the neuronal representations in STS and $\mathrm{PF}$ are learned. We have applied a correlation-based learning rule for the synaptic connections, $a(x, y)$, between any two neurons $x$ and $y$ in the two model layers that is compatible with the field format (for theoretical aspects see Dayan and Abbott (2001); for a discussion in the context of mirror neurons see Keysers and Perrett (2004)):

$$
\tau_{s} \frac{\delta}{\delta t} a(x, y, t)=-a(x, y, t)+\eta f_{2}\left(\bar{u}_{1}(x)\right) f_{2}\left(\bar{u}_{2}(y)\right),
$$

where $\eta>0$ and $\bar{u}_{1}, \bar{u}_{2}$ denote the equilibrium solutions of the relaxation phase in layer STS and layer PF, respectively. Note that the same learning rule is also used to establish the synaptic links between the goal representations and the action means in PF (Erlhagen et al 2006a).

Figure 14(b) shows the stable state of the model dynamics in a goal inference task in which the transporting and placing phase of the action was hidden from view (no suprathreshold activation for the trajectory type in STS). After the learning process, the observation of the IG grip is sufficient to infer the higher target as the end state of the action sequence. The overt behavior (figure 14(c)) shows that the robot uses the previously associated movement primitives PG and AT represented in the action layer F5.

If an observed action is understood, that is, connections between action goal and action means are established, further knowledge associated with that action may be transferred by observing an experienced teacher. The prefrontal cortex (PFC) is commonly believed to extract information about regularities across experiences and to represent rules that can be used to guide action (Miller 2000). We tested in a variation of the basic bridge paradigm the autonomous development of representations that relate object cues to specific goals. Figure 15 illustrates as a simple example the effect of a learned association with object color processed by the vision system. The synaptic links are again established using the correlationbased rule (equation (39)). After a series of observation (and internal simulation) trials, human and robot share the knowledge about what to do with a particular object: a yellow object has to be placed at the higher goal and a blue object at the lower goal. As a consequence, a simple presentation of a blue or yellow object will automatically trigger the associated goal and action means to achieve that goal. This acquired knowledge may then be used in joint action tasks as an additional cue to anticipate the consequences of an ongoing action displayed by the partner.

\section{Discussion}

We presented the theoretical framework of dynamic neural fields as a design tool to build cognitive control architectures for autonomous robots based on neuro-plausible principles. Our close cooperation with experimenters from neuroscience and cognitive science has strongly influenced the proposed architectures for implementing cognitive functions such as goal inference and decision making. They also draw our attention to specific questions which are usually not central to current robotics research such as, for instance, the role of goals in movement observation (Bekkering et al 2000). In general, the architectures reflect the notion that cognitive processes in the brain unfold over time under the influence of multiple internal and external influences (Beer 2000). The choice of neural fields as a mathematical description of population dynamics simplifies or ignores many aspects of processing in the brain (e.g., the timing of spikes). However, the fact that they can be mathematically analyzed is an important advantage when trying to build cognitive agents.

As our robot experiments show, there is a need for some cognitive control of direct sensory-motor schemas (Arkin 1998) which have been proposed in the robotics domain as a means to cope with the dynamics of real-world environments. A local obstacle avoidance behavior as implemented in our dynamic control architecture for the mobile platform, for instance, is not sufficient to guarantee for a successful placing behavior in the bridge paradigm. The existence of the bridge obstacle determines together with the ultimate action goal the type of grasping at the beginning of the action sequence. A 
(a)
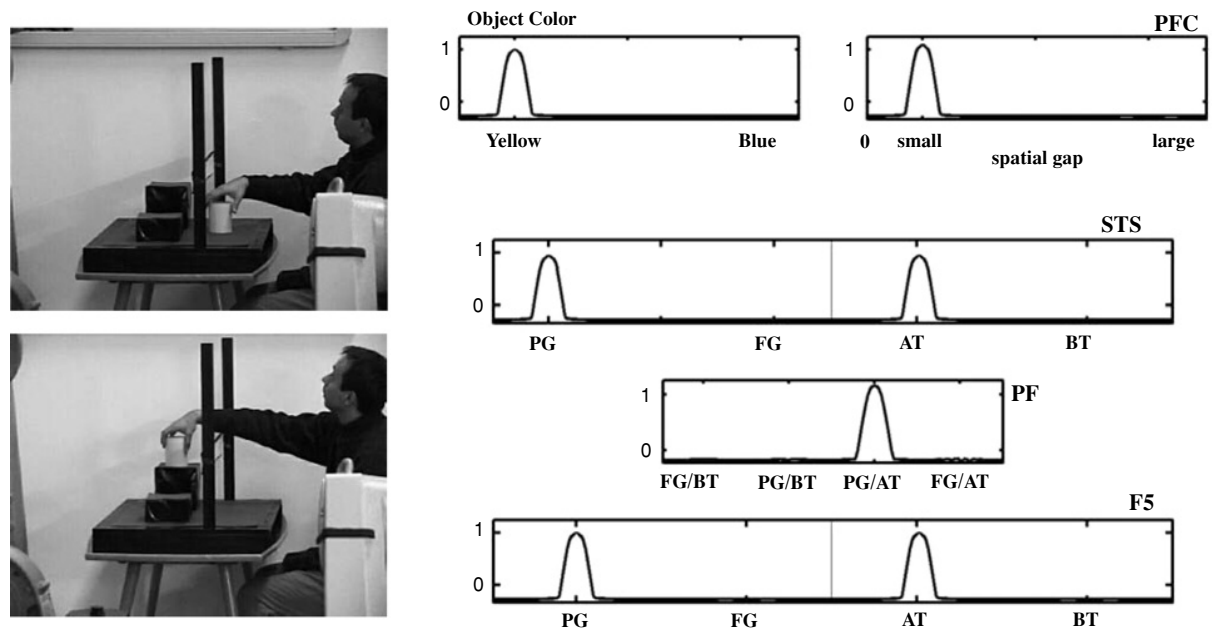

(b)

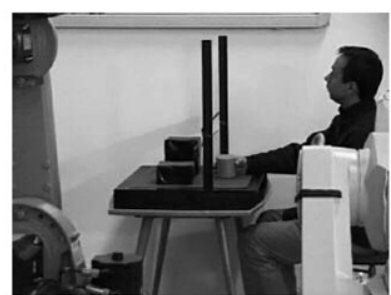

Object Color
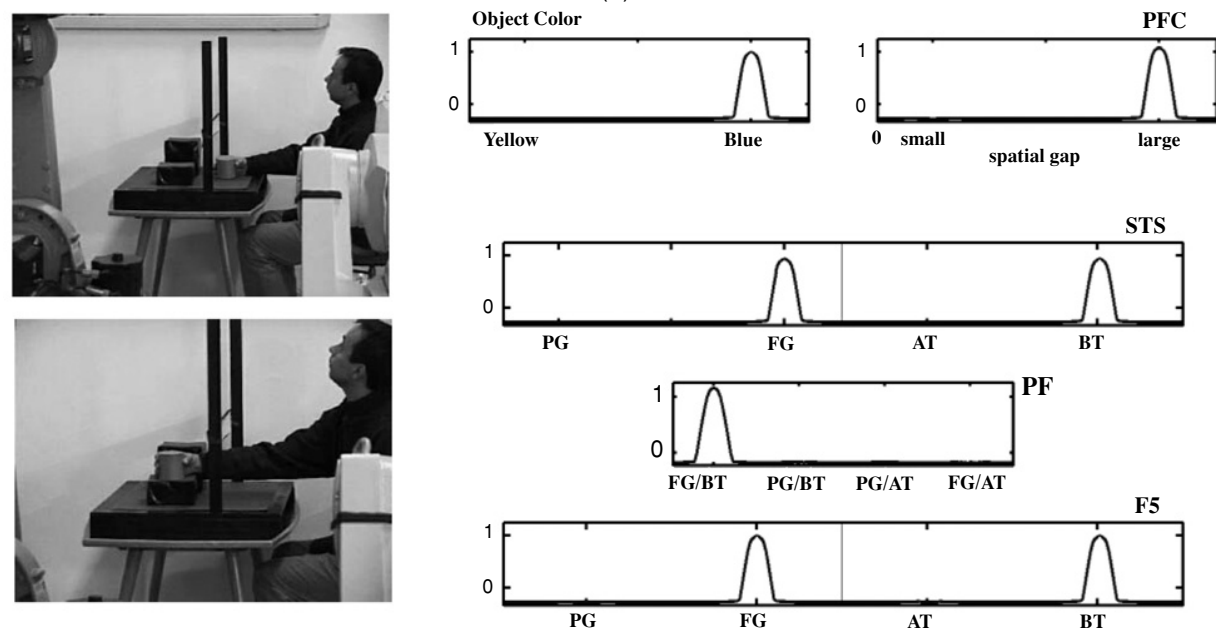

Figure 15. During imitation of a human model, synaptic connections between the neural representation of the action goal and neuronal populations encoding object color are learned. These associations establish a rule: (a) a yellow object has to be placed at the higher goal; (b) a blue object has to be placed at the lower goal. In each case, the stable state of the model dynamics in an imitation experiment is shown. After learning, the cue representation alone may drive the respective goal representation.

wrong initial choice of the grip type (e.g., based on object 'affordances') may thus not be easily compensated on the fly.

It has been argued that cognition in robots would essentially require the capacity to simulate on the basis of internal representations interaction with the environment. Since simulation allows for anticipation, it may constitute an important processing principle to guarantee a fluent real-world behavior (Clark and Grush 1999). The neurophysiological findings summarized in this tutorial strongly support the notion that simulating action and perception is not a centralized process but involves a distributed network of brain areas which are also active during normal perceptual and motor behavior. The fundamental idea for joint action tasks is that these circuits allow an observer to make sense about the partner's motor behavior by linking it to internal representations of goals (e.g., placing an object at a particular position). The anticipated outcome of the action can then be used to choose a complementary goal-directed behavior. As we have shown, coupling various dynamic neural fields each with a specific functionality allows implementing such simulation loops since the internal field representations are self-stabilized. The decision process in the goal layer establishes the proper mappings between sensory inputs, internal states and motor outputs needed to perform a given task. This allows for cognitive control which is necessary whenever prepotent input-output mappings have to be overridden like in the example of the joint search task.

The field architecture implements the fundamental principle that different sources of information compete for expression in behavior (Desimone and Duncan 1995). Relatively small changes in the baseline activity level of neurons due to the integration of contextual information for instance may drastically alter the time course of the pulse evolution. This may in turn alter the processing in 
connected field layers and eventually also the forthcoming behavior (Asaad et al 2000; compare figure 4). A contextdependent interpretation of sensory input is essential for what we recognize as intelligent behavior in autonomous agents.

Although our robots show cognitive behaviors in joint action tasks, they are still not able to autonomously cooperate in completely unstructured and dynamic environments. For instance, the global path planning algorithm for the arm control which we currently employ does not allow us to cope with unpredictable obstacles. Another limitation for our current implementations concerns the vision system. It is yet not powerful enough to autonomously track and classify a sufficiently rich repertoire of body motions. However, for many highly relevant problems there exist already computational models based on dynamic fields or conceptually related recurrent networks which may be adapted for the robotics work. Examples include aspects of higher level vision including the perception of biological motion (Giese 1999, Giese and Poggio 2003), the transformation of retinal to body-centered coordinates (Salinas and Abbott 1995, 1996), the context-dependent selection of visuomotor maps (Salinas 2003), the control of eye movements (Kopecz and Schöner 1995) or the problem of sequence learning and prediction (Abbott and Blum 1996).

Learning defined as the capacity to represent and memorize newly acquired knowledge is in general considered a hallmark of intelligent systems. In distributed field models, learning can be seen as a structural change of the overall dynamical systems which usually evolves over a longer time scale compared to the time scale of a single motor act. Recently, it has been shown that the asymmetry of the weight function which leads to the traveling wave solution in direction space may be autonomously learned during development and practice. The synaptic learning rule utilizes a form of temporal average of recent cell activity to associate the firing of cells encoding directional change (e.g., proprioceptive, visual) with the recent change in the representation of direction (Stringer et al 2002).

The persistent activity patterns in the dynamic fields allow forming associations between events separated in time. Our simple example of learned synaptic links between representations of object cue and goal illustrates that a biologically plausible Hebb rule can be used (see also Salinas and Abbott (1995)). To establish the synaptic links between goal and appropriate action means, the learning process should have access to information related to an evaluation of performance (Erlhagen et al 2006a). Future work should therefore explore in more detail the implementation of unsupervised and reinforcement learning techniques in distributed dynamic field networks (for an overview about mathematical models see Dayan and Abbott (2001)).

In conclusion, recent advances in neuroscience and computational modeling have substantially increased our understanding of the processing principles underlying cognitive functions. The implementation of human-like cognitive skills in robots is still a distant goal. We believe that the dynamic field approach which joins computational, mathematical and engineering efforts is a promising direction to progressively close the gap to the human model.

\section{Acknowledgments}

We would like to thank Albert Mukovskiy for his contributions to the dynamic field model for action understanding and Nzoji Hipólito (financed by the Portuguese and FEDER grant POSI/SRI/38051/2001) for his contributions to the implementation of the search task in physical robots. The present research was conducted in the context of the fp6-IST2 EU-project JAST (project no 003747).

\section{References}

Abbott L F and Blum K I 1996 Functional significance of long-term potentiation for sequence learning and prediction Cereb. Cortex 6 406-16

Amari S 1977 Dynamics of pattern formation in lateral-inhibitory type neural fields Biol. Cybern. 27 77-87

Arkin A C 1998 Behavior Based Robotics (Cambridge, MA: MIT Press)

Asaad W F, Rainer G and Miller E K 2000 Task-specific neural activity in the primate prefrontal cortex J. Neurophysiol. 84 451-9

Baker C I, Keysers C, Jellema T, Wicker B and Perrett D I 2001 Neuronal representation of disappearing and hidden objects in temporal cortex of macaque Exp. Brain Res. 140 375-81

Bastian A, Schöner G and Riehle A 2003 Preshaping and continuous evolution of motor cortical representations during movement preparation Eur. J. Neurosci. 18 2047-58

Beer R 2000 Dynamic approaches to cognitive science Trends Cogn. Sci. 4 91-8

Bekkering H, Wohlschläger A and Gattis M 2000 Imitation of gestures in children is goal-directed Q.J. Exp. Psychol. A 53 153-64

Bergener T, Bruckhoff C, Dahm P, Janssen H, Joublin F, Menzner R, Steinhage A and van Seelen W 1999 Complex behavior by means of dynamical systems for an anthropomorphic robot Neural Netw. 12 1087-99

Bicho E 2000 The Dynamic Approach to Behavior-Based Robotics (Aachen: Shaker)

Bicho E, Mallet P and Schöner G 2000 Target representation on an autonomous vehicle with low-level sensors Int. J. Robot. Res. $19424-47$

Brody C D, Romo R and Kepecs A 2003 Basic mechanisms for graded persistent activity: discrete attractors, continuous attractors, and dynamic representations Curr. Opin. Neurobiol. 13 204-11

Clark A and Grush R 1999 Towards a cognitive robotics Adapt. Behav. 7 5-16

Dayan P and Abbott L F 2001 Theoretical Neuroscience (Cambridge, MA: MIT Press)

Desimone R and Duncan J 1995 Neural mechanism of selective visual attention Annu. Rev. Neurosci. 18 183-222

Douglas R J, Koch C, Mahowald M, Martin K A C and Suarez H H 1995 Recurrent excitation in neocortical circuits Science $\mathbf{2 6 9}$ 981-5

Dusterwitz D, Seamans J K and Sejnowski T J 2000 Neurocomputational models of working memory Nat. Neurosci. 3 1184-91

Engels C and Schöner G 1995 Dynamic fields endow behavior-based robots with representations Robot. Auton. Syst. 14 55-77

Erlhagen W 2003 Internal models for visual perception Biol. Cybern. 88 407-19

Erlhagen W, Mukovskiy A and Bicho E 2006a A dynamic model for action understanding and goal-directed imitation Brain Res. 1083 174-88

Erlhagen W, Mukovskiy A, Bicho E, Panin G, Kiss C, Knoll A, van Schie $\mathrm{H}$ and Bekkering $\mathrm{H}$ 2006b Goal-directed imitation 
for robots: a bio-inspired approach to action understanding and skill learning Robot. Auton. Syst. 54 353-60

Erlhagen W and Schöner G 2002 Dynamic field theory of movement preparation Psychol. Rev. 109 545-72

Eskandar E N and Assad J A 1999 Dissociation of visual, motor and predictive signals in parietal cortex during visual guidance Nat. Neurosci. 2 88-93

Ferrari P F, Rozzi S and Fogassi L 2005 Mirror neurons responding to observation of actions made with tools in monkey ventral premotor cortex J. Cogn. Neurosci. 17 1-15

Fogassi L, Ferrari P F, Gesierich B, Rozzi S, Chersi F and Rizzolatti G 2005 Parietal lobe: from action organization to intention understanding. Science 308 662-7

Gallese V and Goldman A 1998 Mirror neurons and the simulation theory of mind-reading Trends Cogn. Sci. 2 493-501

Giese M A 1999 Dynamic Neural Field Theory for Motion Perception (Boston, MA: Kluwer)

Giese M A and Poggio T 2003 Neural mechanisms for the recognition of biological movements and action Nat. Rev. Neurosci. 4 179-92

Gold J I and Shadlen M N 2002 Banburismus and the brain: the relationship between sensory stimuli, decisions, and reward Neuron 36 299-308

Horta C and Erlhagen W 2006 Robust persistent activity in neural fields with asymmetric connectivity Neurocomputing 69 1141-5

Iossifidis J and Schöner G 2004 Autonomous reaching and obstacle avoidance with the anthropomorphic arm of a robotic assistant using the attractor dynamics approach IEEE 2004 Int. Conf. on Robotics and Automation (26 April-1 May)

Jackson P L and Decety J 2004 Motor cognition: a new paradigm to study self-other interactions Curr. Opin. Neurobiol. 14 259-63

Jellema T and Perrett D I 2002 Coding of visible and hidden actions Attention and Performance: XIX. Common Mechanisms in Perception and Action ed W Prinz and B Hommel (Oxford: Oxford University Press) pp 267-90

Keysers C and Perrett D I 2004 Demystifying social cognition: a Hebbian perspective Trends Cogn. Sci. 8 501-7

Kishimoto K and Amari S 1979 Existence and stability of local excitations in homogeneous neural fields J. Math. Biol. 7 303-18

Kopecz K and Schöner G 1995 Saccadic motor planning by integrating visual information and pre-information on neural, dynamic fields Biol. Cybern. 73 49-60

Laing C R and Troy W C 2003 Two-bump solutions of Amari-type models of neuronal pattern formation Physica D 3-4 190-218

Mel B W 1990 Connectionist Robot Planning: A Neurally-Inspired Approach to Visually-Guided Reaching (New York: Academic)

Menzner R, Steinhage A and Erlhagen W 2000 Generating interactive robot behavior: a mathematical approach From Animals to Animates vol 6, ed J A Meyer, A Berthoz,
D Floreano, H Roitblat and S W Wilson (Cambridge, MA: MIT Press) pp 135-44

Miller E K 2000 The prefrontal cortex and cognitive control Nat. Rev. 1 59-65

Pfeifer R and Scheier C 1999 Understanding Intelligence (Cambridge MA: MIT Press)

Quoy M, Moga S and Gaussier P 2003 Dynamical neural networks for planning and low-level robot control IEEE Trans. Syst. Man Cybern. A 33 523-32

Rittscher J, Blake A, Hoogs A and Stein G 2003 Mathematical modelling of animate and intentional motion Phil. Trans. $R$. Soc. B 358 475-90

Rizzolatti G, Fogassi L and Gallese V 2001 Neurophysiological mechanisms underlying the understanding and imitation of action Nat. Rev. 2 661-70

Salinas E 2003 Background synaptic activity as switch between dynamical states in a network Neural Comput. 15 1439-73

Salinas E and Abbott L F 1995 Transfer of coded information from sensory to motor networks J. Neurosci. 15 6461-74

Salinas E and Abbott L F 1996 A model of multiplicative neural responses in parietal cortex Proc. Natl Acad.Sci. USA 93 11956-61

Schaal S 1999 Is imitation learning the route to humanoid robots? Trends Cogn. Sci. 3 233-42

Schöner G, Dose M and Engels C 1995 Dynamics of behavior: theory and applications for autonomous robot architectures Robot. Auton. Syst. 16 213-45

Shimojo S and Nakayama K 1990 Amodal presence of partially occluded surfaces determines apparent motion Perception 19 285-99

Stringer S M, Trappenberg T P, Rolls E T and Araujo I E T 2002 Self-organizing continuous attractor networks and path integration: one-dimensional models of head-direction cells Netw. Comput. Neural Syst. 13 217-42

Taube J S and Bassett J P 2003 Persistent neural activity in head direction cells Cereb. Cortex 13 1162-72

Taylor J G 1999 Neural bubble dynamics in two dimensions: foundations Biol. Cybern. 80 393-409

Umiltà M A, Kohler E, Gallese V, Fogassi L, Fadiga L, Keysers C and Rizzolatti G 2001 I know what you are doing: a neurophysiological study Neuron 31 155-65

Viola A and Wells W M 1995 Alignment by maximization of mutual information Proc. 5th Int. Conf. on Computer Vision (Cambridge, MA) pp 16-23

Wilson H R and Cowan J D 1973 A mathematical theory of the functional dynamics of cortical and thalamic nervous tissue Kybernetik 13 55-80

Zhang K 1996 Representation of spatial orientation by the intrinsic dynamics of the head-direction cell ensemble: a theory J. Neurosci. 16 2112-26 